\title{
COVID-19 dynamics after a national immunization program in Israel
}

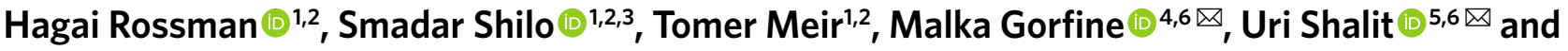 \\ Eran Segal $1,2,6 \bowtie$
}

Studies on the real-life effect of the BNT162b2 vaccine for Coronavirus Disease 2019 (COVID-19) prevention are urgently needed. In this study, we conducted a retrospective analysis of data from the Israeli Ministry of Health collected between 28 August 2020 and 24 February 2021. We studied the temporal dynamics of the number of new COVID-19 cases and hospitalizations after the vaccination campaign, which was initiated on 20 December 2020. To distinguish the possible effects of the vaccination on cases and hospitalizations from other factors, including a third lockdown implemented on 8 January 2021, we performed several comparisons: (1) individuals aged 60 years and older prioritized to receive the vaccine first versus younger age groups; (2) the January lockdown versus the September lockdown; and (3) early-vaccinated versus late-vaccinated cities. A larger and earlier decrease in COVID-19 cases and hospitalization was observed in individuals older than 60 years, followed by younger age groups, by the order of vaccination prioritization. This pattern was not observed in the previous lockdown and was more pronounced in early-vaccinated cities. Our analysis demonstrates the real-life effect of a national vaccination campaign on the pandemic dynamics.

$\Lambda$ $\mathrm{n}$ effective and safe vaccination campaign is urgently needed to halt the rapid spread of severe acute respiratory syndrome coronavirus 2 (SARS-CoV-2) infections and the resulting disease, COVID-19. The BNT162b2 vaccine, developed by BioNTech in cooperation with Pfizer, is a lipid nucleoside-modified RNA encoding the SARS-CoV-2 full-length spike protein ${ }^{1}$. Results from a phase 3 randomized placebo-controlled trial demonstrated that a two-dose regimen in a 21 -d interval conferred $95 \%$ protection against laboratory-confirmed COVID-19 infection in individuals 16 years of age or older'. On 11 December 2020, the Food and Drug Administration issued an Emergency Use Authorization for emergency use of the vaccine for the prevention of COVID-19 (ref. ${ }^{3}$ ), and, after that, an emergency use of the vaccine was also issued by the Israeli Ministry of Health (MOH).

On 20 December 2020, Israel launched a national COVID-19 vaccination campaign ${ }^{4}$, in which BNT162b2 vaccines were administered. The Israeli health system comprises four health maintenance organizations (HMOs), and vaccinations were widely available, according to a prioritization schedule determined by the Israeli $\mathrm{MOH}$. During the early phases of the distribution process, individuals considered as being at high risk for COVID-19 were prioritized for vaccination, including individuals older than 60 years, nursing home residents, healthcare workers and individuals with severe comorbidities. The vaccination campaign was further expanded for individuals aged 55 years and older ${ }^{5}$ and 40 years $^{6}$ and older on 12 January 2021 and 19 January 2021, respectively. On 21 January, individuals aged 16-18 years were also prioritized for vaccination. On 28 January, the vaccination campaign expanded to those aged 35 and older 7 . On 4 February, all individuals aged 16 years and older were eligible to receive the vaccine. However, the HMOs were still instructed to focus their efforts on those aged 50 years and older ${ }^{8}$.
As of 24 February, individuals with a history of severe allergic reactions to the vaccine components, individuals who recovered from COVID-19 or individuals younger than 16 years of age (with the exception of children with severe chronic diseases) were not eligible to receive the vaccine. Starting 3 March 2021, recovered individuals were eligible for a single vaccine dose 3 months after their previous infection date.

The national vaccination campaign has led Israel to having one of the highest rates of vaccinated individuals per capita, with $48.8 \%$, $34 \%$ and $7.5 \%$ of the population having received the first or the second vaccine dose or having recovered from COVID-19, respectively, as of 24 February 2021 , or $68.7 \%, 48 \%$ and $8 \%$, respectively, taking into account population older than 16 years-the population currently eligible for vaccination. In parallel, during the early weeks of the vaccination campaign, the number of cases and hospitalized patients rapidly increased, along with the local emergence of the B1.1.7 variant ${ }^{9}$, leading the government to impose a third lockdown on 8 January 2021. This lockdown was gradually eased starting on 7 February 2021.

When evaluating the effects of vaccines, there are two complementary forms of evaluation. One is measuring the direct effects of the vaccine on the vaccinated individual (termed 'vaccine effectiveness'); the other is measuring the overall effect of the vaccination program on an entire population (termed 'vaccine impact') ${ }^{10}$. On the individual level, preliminary studies from two of the largest Israeli HMOs have attempted to estimate the real-life effectiveness of the vaccine. The first reported an efficacy of $51 \%$ for the first dose after $13-24 \mathrm{~d}^{11}$, whereas the second reported an efficacy of $46 \%$ and $92 \%$ after $14-20 \mathrm{~d}$ from the first dose and 7 or more days from the second dose of the vaccine, respectively ${ }^{12}$. Another report estimated that the vaccine effectiveness is above 95\% 3-4 weeks after the second dose ${ }^{13}$. However, to our

'Department of Computer Science and Applied Mathematics, Weizmann Institute of Science, Rehovot, Israel. ${ }^{2}$ Department of Molecular Cell Biology, Weizmann Institute of Science, Rehovot, Israel. ${ }^{3}$ Pediatric Diabetes Clinic, Institute of Diabetes, Endocrinology and Metabolism, Rambam Health Care Campus, Haifa, Israel. ${ }^{4}$ Department of Statistics and Operations Research, Tel Aviv University, Ramat Aviv, Israel. ${ }^{5}$ Technion-Israel Institute of Technology, Haifa, Israel. ${ }^{6}$ These authors contributed equally: Malka Gorfine, Uri Shalit, Eran Segal. ${ }^{凶}$-mail: gorfinem@tauex.tau.ac.il; urishalit@technion.ac.il; 


\section{Table 1 | Policy summary}

Background

COVID-19 vaccinations are one of the most promising prevention measures to mitigate the spread of the devastating COVID-19 pandemic. Although vaccine effectiveness has been demonstrated in clinical trials and using real-world data, the effect of vaccination campaigns at the population level, which is also dependent on additional factors that can influence disease transmission, has not yet been studied.

Main findings and

Here we show the real-life effect of a mass vaccination campaign with stepped prioritization on the dynamics of the COVID-19

limitations pandemic in Israel. We observed a large and early decrease in COVID-19 cases and hospitalizations in individuals older than 60 years, followed by younger age groups, according to the order of vaccination prioritization. The fast and early decline of cases in older individuals was more pronounced in early-vaccinated cities compared to late-vaccinated cities. Although a third national lockdown was implemented concurrently with the vaccination campaign, these profound effects were not observed in the previous national lockdown. One limitation is that the effects of the vaccination campaign observed here could be influenced by factors specific to Israel and its healthcare system. Financial and regional disparities in other healthcare systems might affect the distribution and availability of vaccinations, thereby influencing pandemic dynamics differently.

Policy implications Our results indicate that the vaccination campaign in Israel has had a substantial effect on the dynamics of the COVID-19 pandemic. As Israel is one of the first countries to implement a vaccination campaign of this scale, our findings have generalizable public health implications and support the effectiveness of nationwide vaccination campaigns.

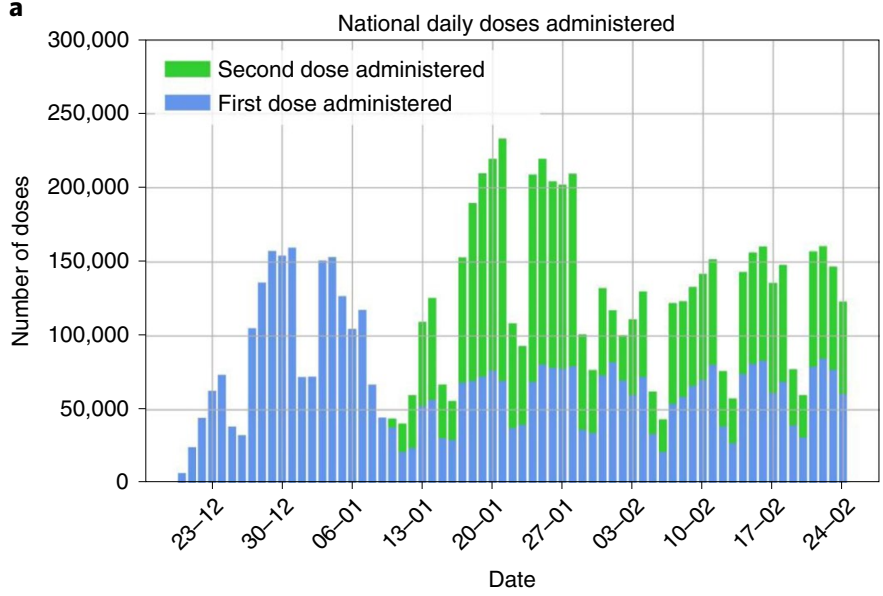

b

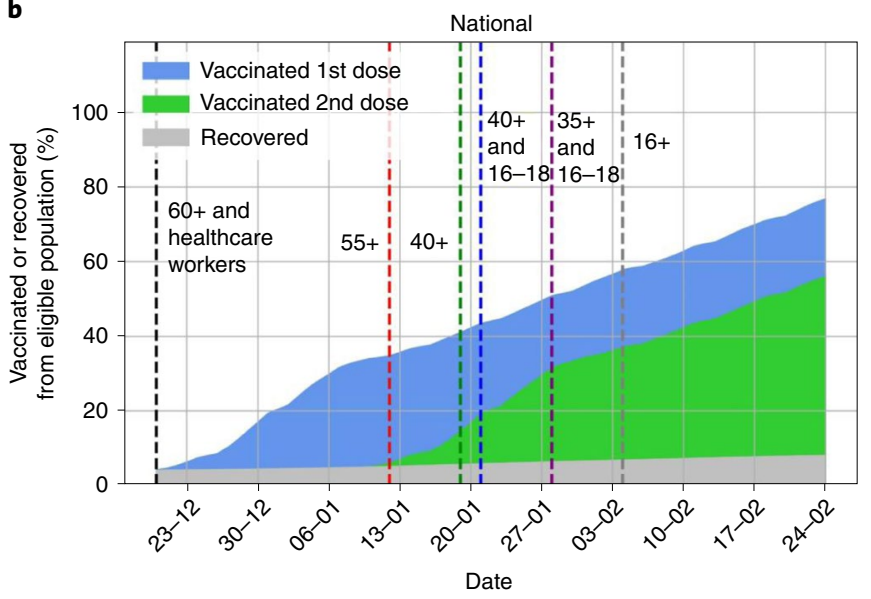

C 0

20

40

60

80

100

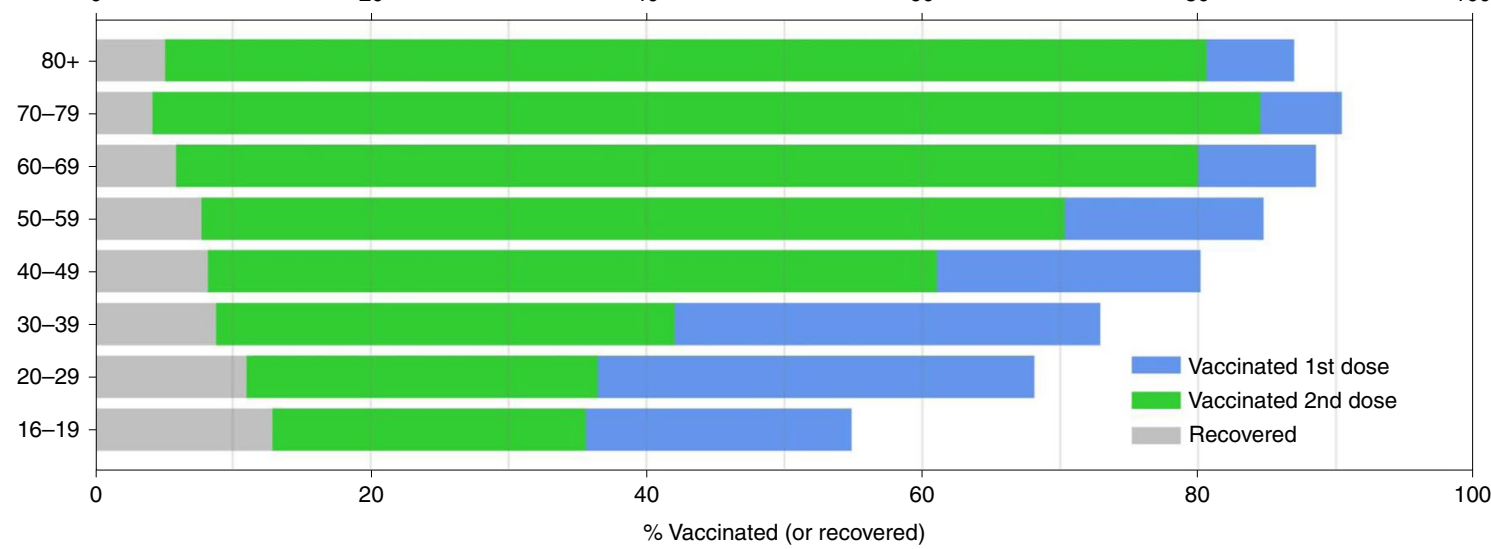

Fig. 1 | National vaccination numbers in Israel. a, Daily national number of administered vaccination doses: first dose (blue bars) and second dose (green bars). b. Cumulative percentage of the national population recovered or vaccinated. The vaccinated population that received the first dose is shown in blue; the vaccinated population that received the second dose is shown in green; the recovered population is shown in gray. Times at which age groups were formally eligible for vaccination are shown as dashed vertical lines. c, Vaccinated or recovered percentage per age group on 24 February 2021.

knowledge, very few studies ${ }^{14}$ thus far have analyzed the effect of the vaccination campaign on the patterns of pandemic dynamics at the population level. As Israel is one of the first countries to implement a vaccination campaign of this magnitude, we think that this quantification might be of interest to many countries worldwide. A summary of the main findings and limitations of the study is provided in Table 1.

\section{Results}

Starting on 20 December 2020-the initiation of the vaccination campaign - the number of vaccines administered per day began at approximately 50,000 , quickly rose to over 150,000 by 24 December and reached a maximum of 231,010 vaccinations on 21 January 2021 (Fig. 1). Vaccinations per day for each age group are shown in 

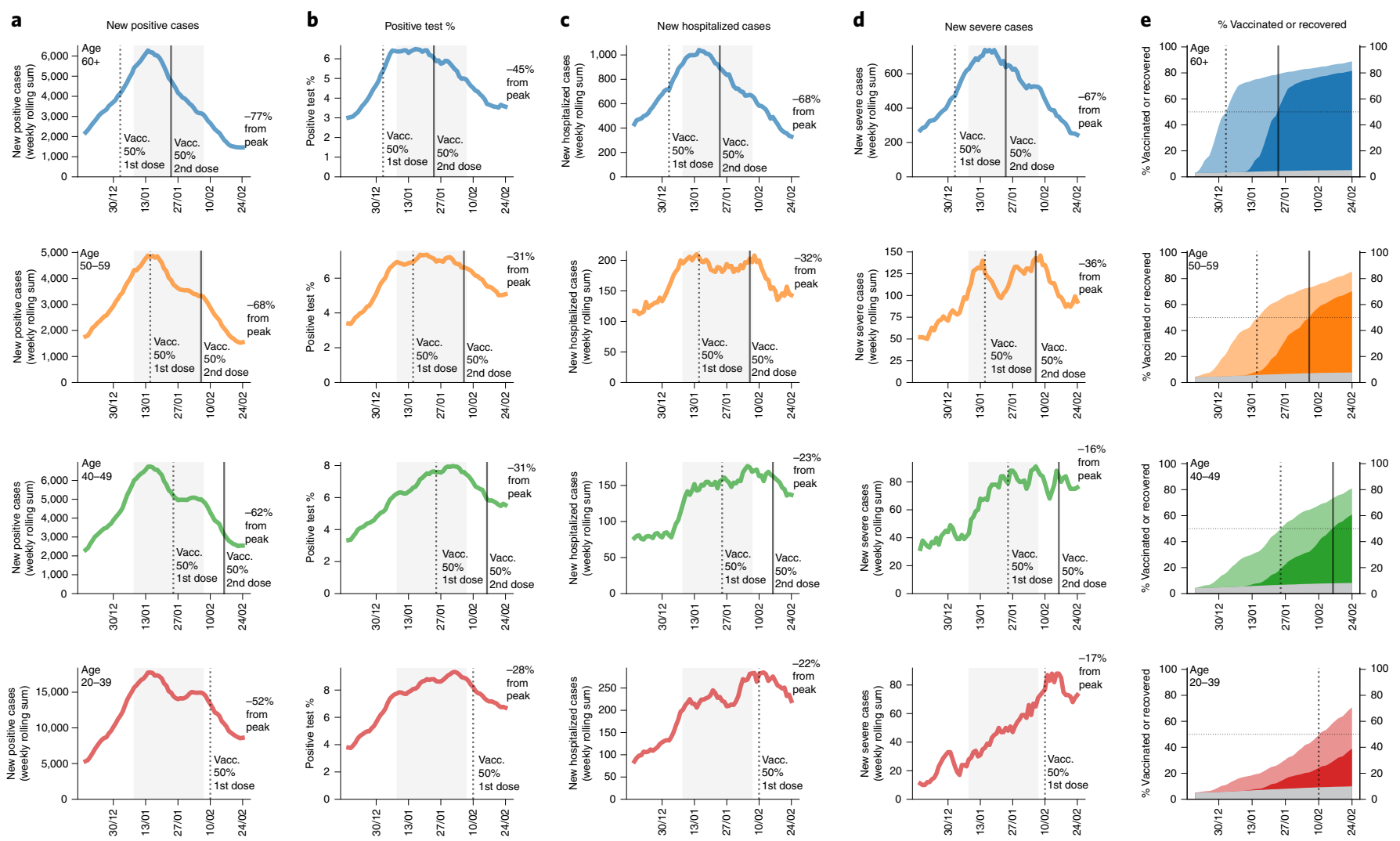

Fig. 2 | Dynamics of national clinical measures for different age groups: first row (blue)-aged over 60 years; second row (orange)-aged 50-59 years; third row (green)-aged 40-49 years; fourth row (red)-aged 20-39 years. a, Rolling weekly sum of new positive PCR cases. $\mathbf{b}$, Percent of PCR tests that come out positive. c, Rolling weekly sum of new hospitalizations. d, Rolling weekly sum of new severe hospitalizations. e, Cumulative vaccinated population percentage per age group. Light color represents the population percentage that has been vaccinated with a first dose; dark coloring represents the population percentage per age group that has been vaccinated with a second dose; gray coloring represents the recovered population percentage per age group (not eligible for vaccination). In all figures, the date at which $50 \%$ of the age group population was vaccinated by a first dose or recovered is shown as a black dotted vertical line, and the date at which $50 \%$ of the population was vaccinated with a second dose or recovered is shown as a black vertical line. In a-d, the lockdown time period is displayed as a gray filling, and the percentage reduction with respect to the peak value of the last date (24 February 2021) is presented at the tail of each curve. An aggregate version of the data presented in Fig. $2 \mathrm{c}$ and 2 e was previously published ${ }^{22}$. A summary of this figure is shown in Supplementary Table 1.

Extended Data Fig. 1. By 7 January 2021, almost 70\% of the population over 60 years old had already been vaccinated (first dose) or recovered, increasing gradually to $88.5 \%$ (first dose) and $81 \%$ (both doses) by 24 February. Cumulative percentages of recovered or vaccinated individuals by age group are shown in Extended Data Fig. 2.

The temporal changes in the number of new COVID-19 cases and hospitalizations in Israel from 18 December 2020 to 24 February 2021 are summarized in Figs. 2 and 4. Several days after the initiation of the lockdown on 8 January 2021 and the beginning of the administration of the second vaccine doses on 10 January, the number of new COVID-19 cases in individuals aged 60 years and older reached a peak. This peak was followed by a peak in mild, moderate or severe hospitalization of individuals and a later peak in severe hospitalization of the same age group. Between 15 January and 24 February, the number of new cases and hospitalizations in this age group declined. As several potential factors other than the vaccines could have influenced this decline, we aimed to estimate the specific contribution of the vaccination campaign to the observed patterns.

We first compared individuals 60 years and older, who were prioritized to receive the vaccine earlier, to younger individuals (Fig. 2 and Supplementary Table 1). The decrease in the number of cases and hospitalizations was larger and earlier in individuals 60 years and older compared to younger individuals. Moreover, the decrease in these clinical measures was according to the order of real-life vaccination rate (Extended Data Fig. 3), which was guided by the prioritization schedule from the Israeli $\mathrm{MOH}$. For example, a decrease of $45 \%$ versus $28 \%$ in the percentage of positive tests and $68 \%$ versus $22 \%$ in hospitalizations compared to the peak was observed in individuals 60 years and older compared to individuals aged $20-39$ years. This is also evident in a considerable shift toward younger ages in the distribution of ages of patients with severe COVID-19 (Fig. 4). To allow youth to attend their school exams, adolescents (age 16-18 years) were also prioritized early in the vaccination campaign. Many young adults (age 18-21 years) serving in the Israel Defense Forces were also prioritized. Notably, COVID-19 cases and the positivity rates of diagnostic tests dropped more rapidly in this group (age 16-21 years) compared to similar age groups that were not vaccinated yet (14-15 years and 22-23 years) (Extended Data Fig. 4).

Figure 3 presents a set of comparisons of clinical measures between two time periods: a period after the second national lockdown implemented by the Israeli government on 18 September 2020 (Fig. 3a-d), and after the third national lockdown, implemented on 8 January 2021 (Fig. 3a-d). Although all clinical measures had almost the same dynamics for all age groups during the first period (second lockdown), these dynamics differed substantially across age groups in the second period (third lockdown + vaccine drive), 
a
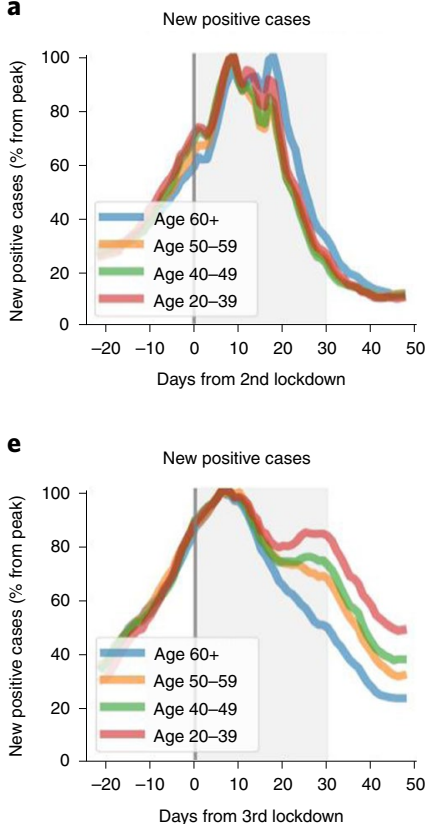

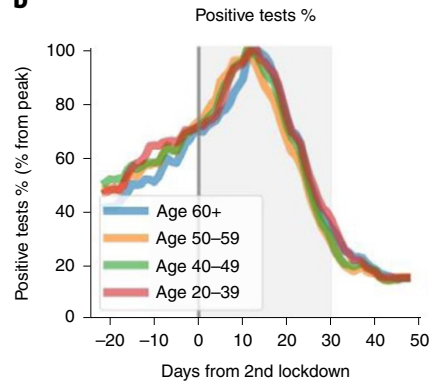

f

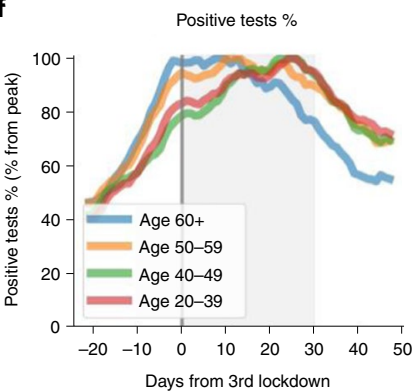

i
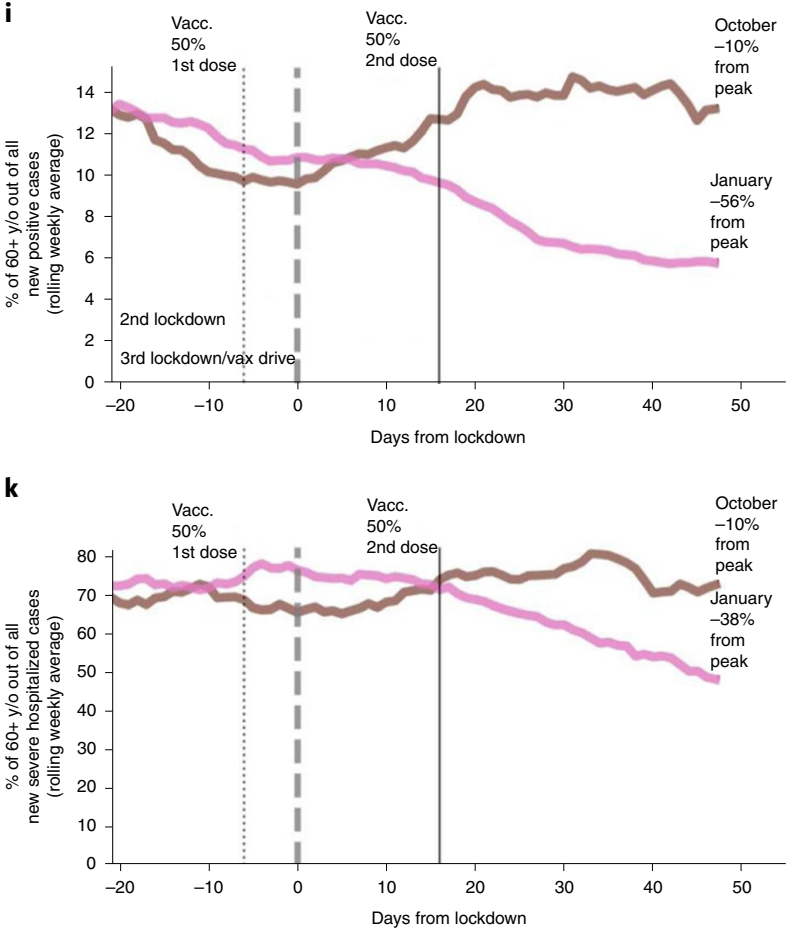

c
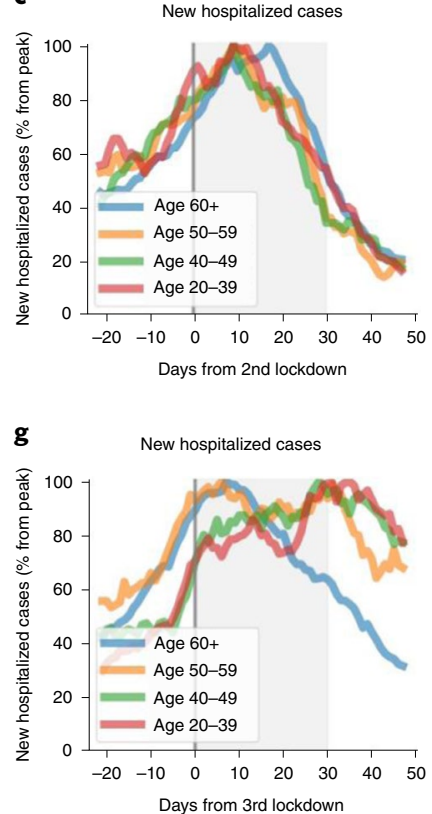

d
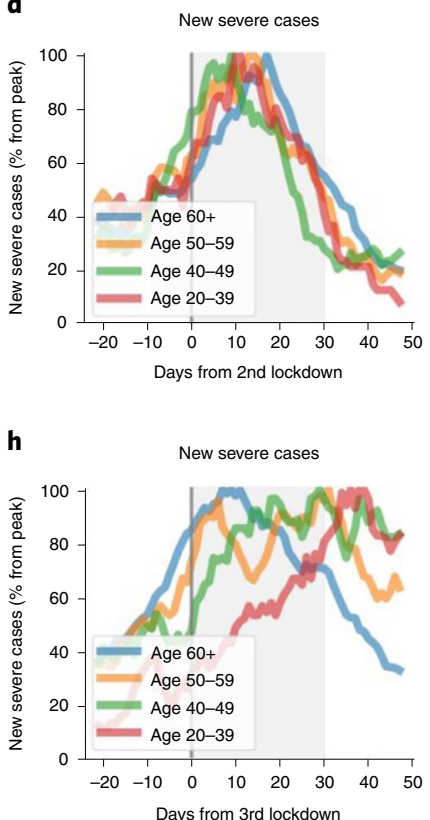
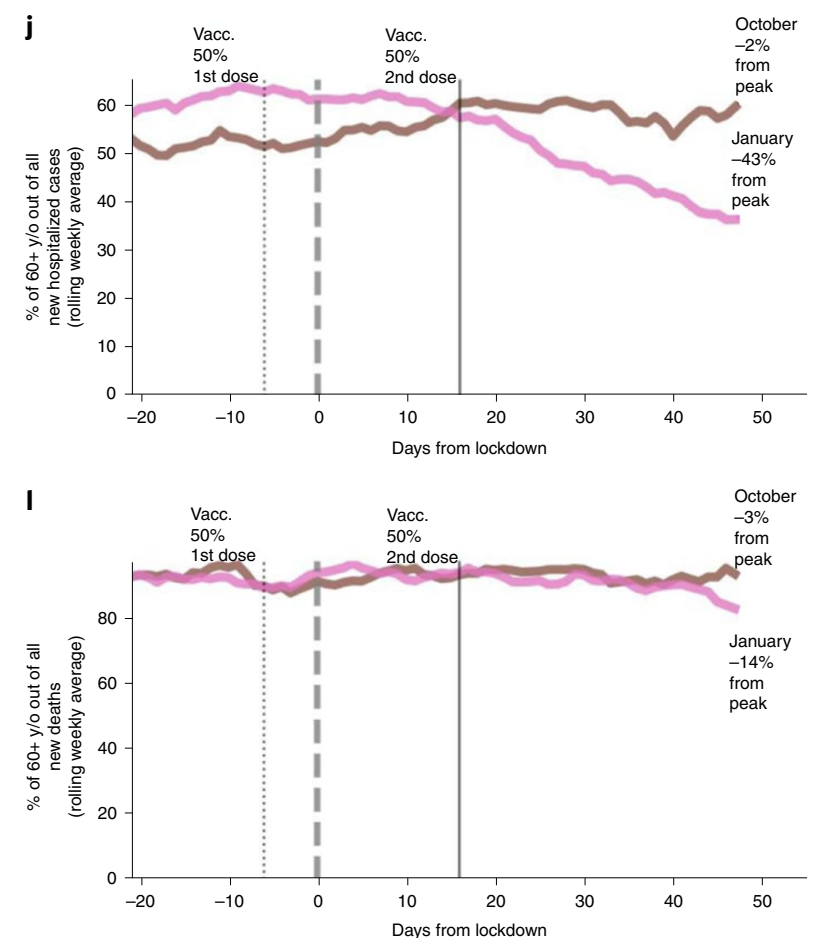

Fig. 3 | Comparison across age groups and lockdowns. Comparison among age groups 20-39 (red), 40-49 (green), 50-59 (orange) and over 60 (blue) years. a, Percent drop of new positive cases from peak value at the time period around the second lockdown. $\mathbf{b}$, Percent drop of the percent of PCR tests that are positive, relative to the peak at the time period around the second lockdown. c, Percent drop of new hospitalizations from the peak at the time period around the second lockdown. d, Percent drop of new severe hospitalizations from the peak at the time period around the second lockdown. e, Percent drop of new positive cases from peak value at the time period around the third lockdown. f, Percent drop of the percent of PCR tests that are positive, relative to the peak at the time period around the third lockdown. $\mathbf{g}$, Percent drop of new hospitalizations from the peak at the time period around the third lockdown. h, Percent drop of new severe hospitalizations from the peak at the time period around the third lockdown. In a-d, 'Day 0' represents the second lockdown start date: 18 September 2020. In e-h, 'Day O' represents the third lockdown start date: 8 January 2021. In a-h, the lockdown time period is displayed as a gray filled area. $\mathbf{i}-\mathbf{I}$ show the percent of age group $60+$ years old out of the population of $\mathbf{i}$; new positive cases j; new hospitalizations $\mathbf{k}$; new severe hospitalizations I. New deaths. The percentage around the second lockdown is shown in brown, and the percentage around the third lockdown is shown in pink. The day on which $50 \%$ of the population received the first dose or recovered is displayed as a dotted black vertical line, and the day on which $50 \%$ of the population received the second dose or recovered is displayed as a black vertical line (relevant with respect to the third lockdown only). y/o, year-olds. 


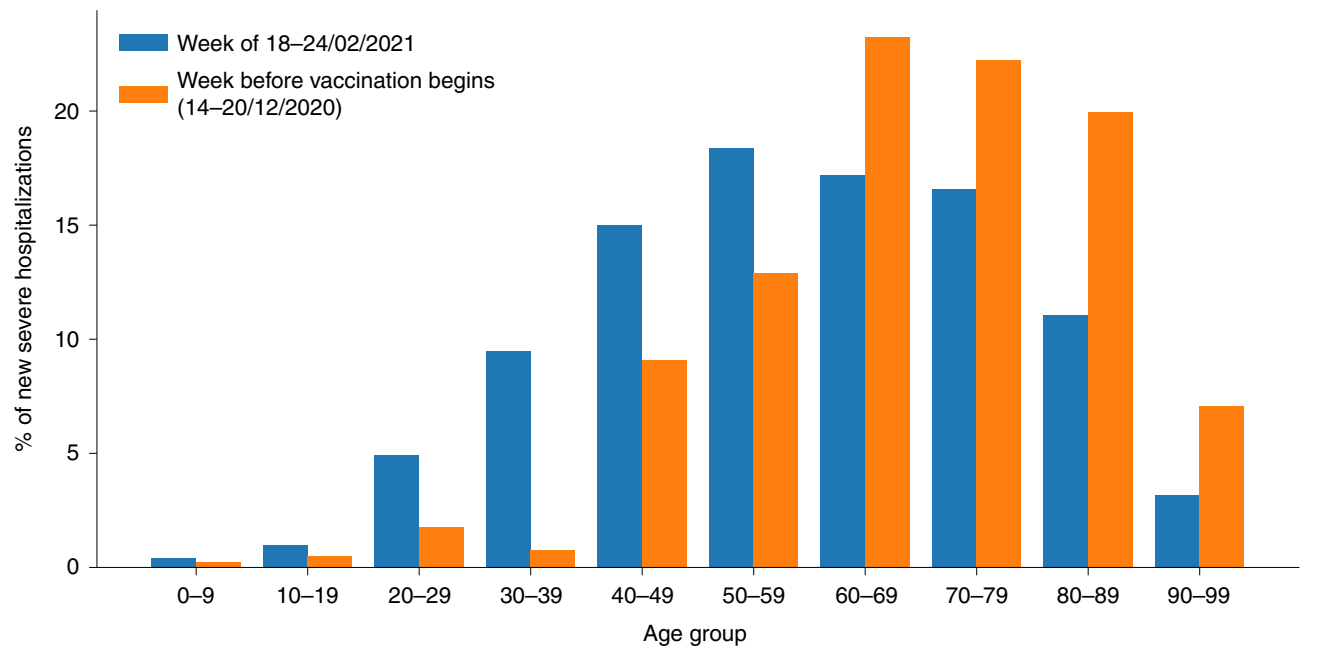

Fig. 4 | Severe hospitalizations age distributions. Percentage of each age group of all new severe hospitalizations at two different weeks: the week before the vaccination campaign initiated (orange) and the week of 18-24 February 2021 (blue).

where a larger and earlier decline in older individuals (older than 60 years) compared to younger individuals was apparent.

The analysis between early-vaccinated cities and late-vaccinated cities (Methods) revealed a larger and earlier decrease in the number of COVID-19 cases and hospitalizations of individuals 60 years and older in cities vaccinated early compared to late-vaccinated cities (Fig. 5 and Supplementary Table 2). For example, in early-vaccinated cities, there was a decrease of $88 \%$ in cases and of $79 \%$ in severe hospitalizations compared to peak values, whereas, in late-vaccinated cities, a smaller decrease of $78 \%$ in cases and $66 \%$ in severe hospitalizations was observed. Finally, to obtain a higher geographic resolution, we compared between early-vaccinated geographical statistical areas (GSAs) and late-vaccinated GSAs (Methods, Extended Data Fig. 5 and Supplementary Table 3). This analysis revealed similar findings as the city-level analysis.

\section{Discussion}

Here we show early signs for the effect of a national vaccination campaign in Israel on the pandemic dynamics. Our analysis revealed that a little over 2 months after the initiation of the vaccination campaign, with $85 \%$ of individuals older than 60 years already vaccinated with two doses (24 February 2021), there was an approximately $77 \%$ drop in cases, a $45 \%$ drop in positive test percentage, a $68 \%$ drop in hospitalizations and a $67 \%$ drop in severe hospitalizations compared to peak values. Although multiple other factors besides the vaccines might have influenced these results, several observations suggest that these patterns are likely to be driven, to a considerable degree, by the vaccines. First, the decline in the measures above is greater in individuals 60 years and older who were prioritized to receive the vaccine earlier, with consecutive drops observed in younger age groups later, according to the order of vaccine prioritization, including earlier drops in some young age groups (16-21 years) prioritized over older age groups (21-35 years). Moreover, the decrease in the clinical measures that we analyzed occurred only after more than $50 \%$ of the population in a given age group had been vaccinated by the first dose or recovered (Fig. 2). Second, the effect was greater in cities and GSAs where a higher fraction of individuals were vaccinated earlier. Finally, we did not observe a similar pattern of a larger and faster decline of cases and hospitalizations in older individuals during the previous lockdown implemented in Israel (between 18 September 2020 and 18 October 2020), in which all clinical measures had similar dynamics in all age groups (Fig. 3 and Extended Data Figs. 3-7).
Although previous reports, both in a clinical trial setting ${ }^{2,11}$ and on real-life data ${ }^{12}$, have indicated efficacy of the vaccine after the first dose, the improvement in the number of hospitalized patients in Israel was evident approximately 3-4 weeks after initiation of the vaccination campaign. We think that this has several explanations. First, the real-life effectiveness might be different from the efficacy reported in the clinical trial. In particular, the logistics of refrigeration, storage, transportation and on-site administration of the vaccines in real-world settings and during a rapid deployment campaign could have been imperfect, thus lowering effectiveness. Second, the effect might be heterogeneous and population dependent. For example, it is possible that older individuals, who were prioritized earlier in the vaccination campaign, could have a reduced or belated response to the vaccination due to a deterioration in both innate and adaptive immune function, also termed immunosenescence, as was previously shown for other vaccines ${ }^{15,16}$. Third, it is possible that the effectiveness of the vaccine is reduced in light of the emergence of new strains, such as the B.1.1.7 variant ${ }^{17}$ (which was the dominant strain in Israel in February 2021 ${ }^{18}$ ) and the $501 . \mathrm{V} 2$ variant $^{19}$, which might be associated with an increased risk of death ${ }^{20}$.

Even if the real-life effectiveness of the vaccine is similar to the efficacy reported in the clinical trial, as shown by Dagan et al. ${ }^{12}$, the effect of the vaccination program on the population as a whole depends on additional factors, including the national vaccine coverage, vaccine allocation among different subgroups of individuals and social mixing of the different groups that could affect disease transmission through indirect effects. It is also possible that vaccinated individuals might alter their behavior (for example, mobility) and decrease adherence to public health prevention guidance (for example, physical distancing and face masks), thereby increasing viral transmission. Notably, mobility data in different districts in Israel throughout the study period (Extended Data Fig. 8) show that mobility was influenced mainly by the restrictions imposed during the lockdown and the loosening of these restrictions thereafter. As expected by Israel's small geographic size, the variation between different districts was relatively small, and it was difficult to ascertain if it was affected by the small variation in the vaccination rate. Future studies are, therefore, needed to assess the effects of the vaccination campaign on different aspects of human behavior.

Another consideration is that viral transmission could also occur in the vaccination sites themselves. The vaccination sites should be large and ventilated to decrease the probability of transmission on 

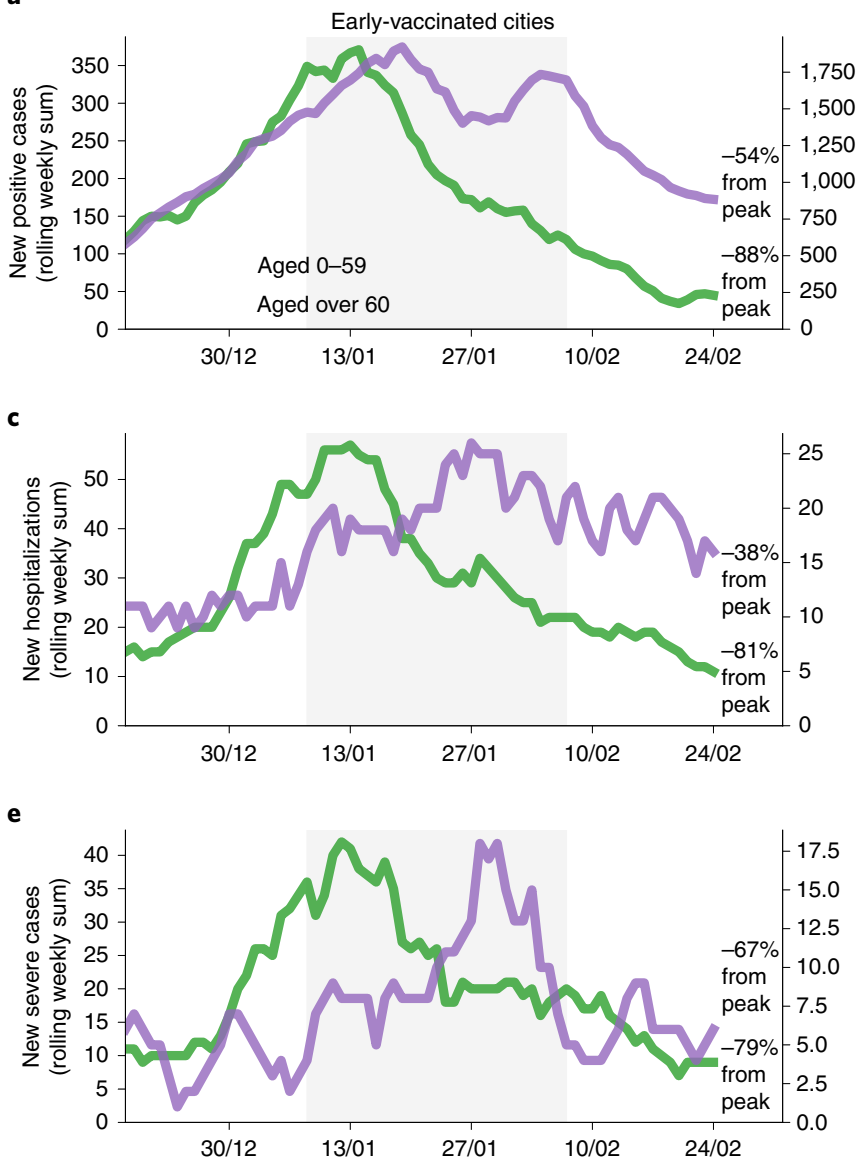

g

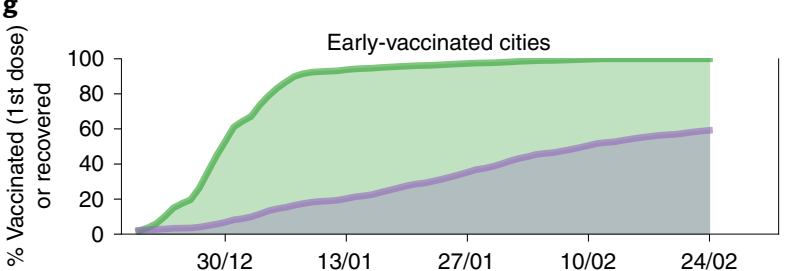

b

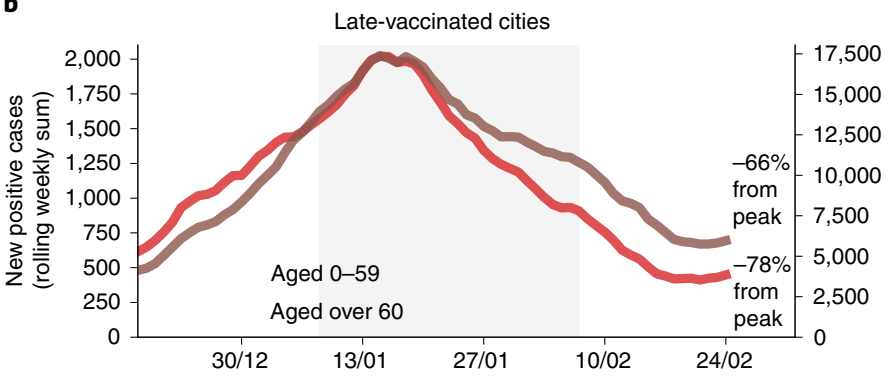

d

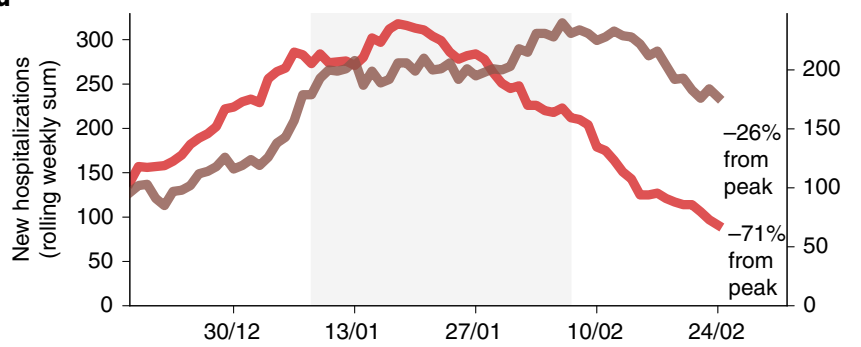

f

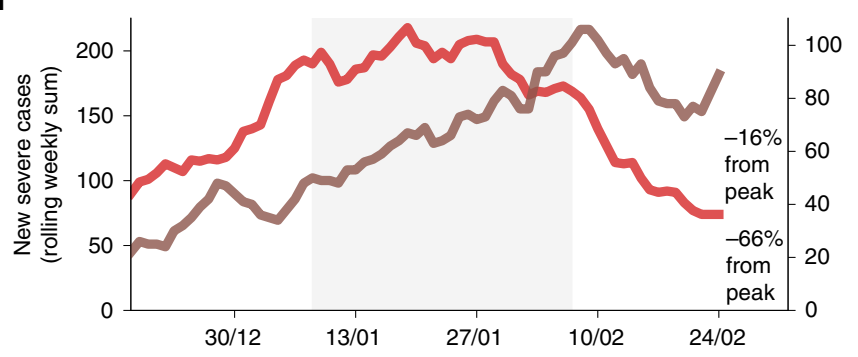

h

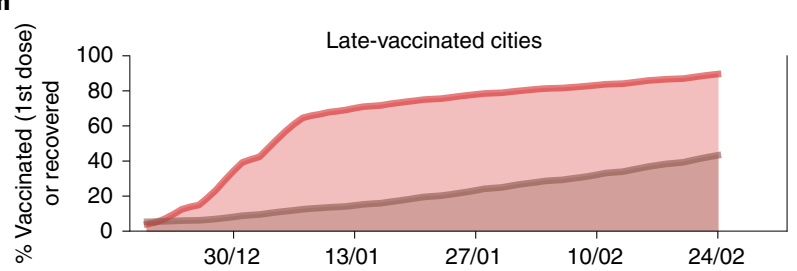

Fig. 5 | Comparison between age groups 0-59 years and 60+ years from cities with most of the population vaccinated early and cities with most of the population vaccinated late. All figures are for the time period of 18 December 2020 to 24 February 2021. In a-f, the lockdown time period is shown as gray filling. Note: a-f are presented with two different $y$-axis scales to highlight relative within-population trends. Age group 0 -59 years is shown as a purple line in $\mathbf{a}, \mathbf{c}$ and $\mathbf{e}$ and as a brown line in $\mathbf{b}, \mathbf{d}$ and $\mathbf{f}$. Age group 60+ is shown as a green line in $\mathbf{a}, \mathbf{c}$ and $\mathbf{e}$ and as a red line in $\mathbf{b}, \mathbf{d}$ and $\mathbf{f}$. $\mathbf{a}$, Rolling weekly sum of new positive cases in early-vaccinated cities. b. Rolling weekly sum of new positive cases in late-vaccinated cities. c, Rolling weekly sum of new mild, moderate or severe hospitalizations in early-vaccinated cities. d, Rolling weekly sum of new mild, moderate or severe hospitalizations in late-vaccinated cities. e, Rolling weekly sum of new severe hospitalizations in early-vaccinated cities. f, Rolling weekly sum of new severe hospitalizations in late-vaccinated cities. $\mathbf{g}$, Cumulative percentage of the population recovered or vaccinated (first dose) in early-vaccinated cities. Age group $60+$ is shown as a green line; age group 0-59 is shown as a purple line. $\mathbf{h}$, Cumulative percentage of the population recovered or vaccinated (first dose) in late-vaccinated cities. Age group 60+ is shown as a red line; age group 0-59 is shown as a brown line. A summary of this figure is shown in Supplementary Table 2.

site. Finally, there is a clear pattern where areas in Israel with higher infection rates and a lower socioeconomic status have lower vaccination rates, despite widespread vaccine availability ${ }^{21}$.This trend might also diminish or delay the overall effect of the campaign as those who are at a higher risk of being infected are less vaccinated. Further efforts should, therefore, be made to encourage these populations to vaccinate and make the vaccines even more easily accessible to them. We note that exact individual-level efficacy numbers cannot be deduced from our analysis, and that, owing to all of the above issues, our results might be consistent with efficacies that are either lower or higher than those reported in the original clinical trial.
Our study had several limitations. First, it was an observational study as opposed to a randomized clinical trial, and, therefore, causal effects are difficult to infer. Second, the comparison between the second and third lockdown might be influenced by factors such as the total number of COVID-19 cases in the beginning of each lockdown, testing policy, hospitalization policy and public compliance with the restrictions that could have changed with time. Similarly, differences among cities might be influenced by behavioral and social differences beyond availability of vaccines. However, none of these factors was likely to cause the different patterns observed in the different age groups reported here. Finally, the effects of the vaccination campaign observed here could be influenced by factors 
specific to Israel and its healthcare system, in which all citizens are mandated to join one of the official non-profit health insurance organizations. Financial and regional disparities in other healthcare systems might affect the distribution and availability of vaccinations, thereby influencing the real-life efficacy of the vaccines.

Overall, we show an analysis of large-scale, real-world data from Israel demonstrating real-life effectiveness of a national vaccination campaign. Although our findings are preliminary, they have major public health implications for the struggle against the COVID-19 pandemic. More studies aimed at assessing the effectiveness of the vaccination on reducing the transmission of SARS-CoV-2 are needed, both on the individual level and on the population level, with larger longitudinal follow-up and in additional populations.

\section{Online content}

Any methods, additional references, Nature Research reporting summaries, source data, extended data, supplementary information, acknowledgements, peer review information; details of author contributions and competing interests; and statements of data and code availability are available at https://doi.org/10.1038/ s41591-021-01337-2.

Received: 2 March 2021; Accepted: 5 April 2021;

Published online: 19 April 2021

\section{References}

1. Walsh, E. E. et al. Safety and immunogenicity of two RNA-based Covid-19 vaccine candidates. N. Engl. J. Med. 383, 2439-2450 (2020).

2. Polack, F. P. et al. Safety and efficacy of the BNT162b2 mRNA Covid-19 vaccine. N. Engl. J. Med. 383, 2603-2615 (2020).

3. Tanne, J. H. Covid-19: FDA panel votes to approve Pfizer BioNTech vaccine. BMJ 371, m4799 (2020).

4. Rosen, B., Waitzberg, R. \& Israeli, A. Israel's rapid rollout of vaccinations for COVID-19. Isr. J. Health Policy Res. 10, 6 (2021).

5. Ministry of Health. Starting Tuesday (12.1.21), insured HMO members 55 years old or older will be vaccinated. https://www.gov.il/en/departments/ news/11012021-01

6. Ministry of Health. Ministry of Health Director General ordered to vaccinate 40 year olds and older. https://www.gov.il/en/departments/news/19012021-02 (19 January 2021).

7. Ministry of Health. Back to Life: Vaccines for Coronavirus. https://govextra. gov.il/ministry-of-health/covid19-vaccine/home-en/?gclid=Cj0KCQiA6t6ABh DMARIsAONIYyw5SIeaB9_2PcjHXz6rvNrFittCUuIG9tqa0Aqq7IEXvBizn xo0-kAaAvDvEALw wcB
8. Ministry of Health. Operation 'Hozrim LeHayim' expands. https://www.gov.il/ en/departments/news/03022021-01

9. Ministry of Health. 18 positive samples of the English variant ('The British Mutation') of the coronavirus detected. https://www.gov.il/en/departments/ news/01012021-01

10. Hanquet, G., Valenciano, M., Simondon, F. \& Moren, A. Vaccine effects and impact of vaccination programmes in post-licensure studies. Vaccine 31, 5634-5642 (2013).

11. Chodcik, G. et al. The effectiveness of the first dose of BNT162b2 vaccine in reducing SARS-CoV-2 infection 13-24 days after immunization: real-world evidence. Preprint at https://www.medrxiv.org/content/10.1101/2021.01.27.212 50612v1 (2021).

12. Dagan, N. et al. BNT162b2 mRNA Covid-19 vaccine in a nationwide mass vaccination setting. N. Engl. J. Med. https://doi.org/10.1056/NEJMoa2101765 (2021).

13. Aran, D. Estimating real-world COVID-19 vaccine effectiveness in Israel using aggregated counts. Preprint at https://www.medrxiv.org/content/10.1101 /2021.02.05.21251139v2 (2021)

14. De-Leon, H., Calderon-Margalit, R., Pederiva, F., Ashkenazy, Y. \& Gazit, D. First indication of the effect of COVID-19 vaccinations on the course of the COVID-19 outbreak in Israel. Preprint at https://www.medrxiv.org/content/10 $.1101 / 2021.02 .02 .21250630 \mathrm{v} 1$ (2021).

15. Lang, P.-O., Govind, S., Mitchell, W. A., Siegrist, C.-A. \& Aspinall, R. Vaccine effectiveness in older individuals: what has been learned from the influenza-vaccine experience. Ageing Res. Rev. 10, 389-395 (2011).

16. Fisman, D. N., Agrawal, D. \& Leder, K. The effect of age on immunologic response to recombinant hepatitis B vaccine: a meta-analysis. Clin. Infect. Dis. 35, 1368-1375 (2002)

17. Wise, J. Covid-19: new coronavirus variant is identified in UK. BMJ 371, m4857 (2020).

18. Munitz, A., Yechezkel, M., Dickstein, Y., Yamin, D. \& Gerlic, M. The rise of SARS-CoV-2 variant B.1.1.7 in Israel intensifies the role of surveillance and vaccination in elderly. Preprint at https://www.medrxiv.org/content/10.1101/2 021.02.16.21251819v1 (2021).

19. Health Ministry says South African virus strain found in Israel for 1st time. The Times of Israel https://www.timesofisrael.com/

health-ministry-says-south-african-virus-strain-found-in-israel-for-1st-time/ (9 January 2021).

20. Iacobucci, G. Covid-19: new UK variant may be linked to increased death rate, early data indicate. $B M J \mathbf{3 7 2}, \mathrm{n} 230$ (2021).

21. Caspi, G. et al. Socioeconomic disparities and COVID-19 vaccination acceptance: experience from Israel. Preprint at https://www.medrxiv.org/conte nt/10.1101/2021.01.28.21250716vl (2021).

22. Shilo, S., Rossman, H. \& Segal, E. Signals of hope: gauging the impact of a rapid national vaccination campaign. Nat. Rev. Immunol. 21, 198-199 (2021).

Publisher's note Springer Nature remains neutral with regard to jurisdictional claims in published maps and institutional affiliations.

(c) The Author(s), under exclusive licence to Springer Nature America, Inc. 2021 


\section{Methods}

A retrospective analysis was conducted on data originating from the Israeli $\mathrm{MOH}$ from 28 August 2020 to 24 February 2021. The data included information on age, sex, date of positive SARS-CoV-2 polymerase chain reaction (PCR) test, date of hospitalization, clinical state during hospitalization and date of death or recovery for each individual. Data on national vaccination are available online (http://data. gov.il/dataset/covid-19/) and include the number of daily vaccine doses, separated to first and second doses, administered in each city by age groups (a scale of 10 years). Recovered individuals were included in the vaccinated group in all of the analyses, as previous studies analyzing the antibody responses after recovery showed persistent antibody titers against the SARS-CoV-2 spike protein for several months after infection ${ }^{23}$. Throughout the study period, these individuals were not eligible to receive the vaccine. A total of 3,210,200 individuals vaccinated with both doses of the vaccine and 711,949 recovered individuals were included in the analysis. An exemption from institutional review board approval was determined by the Israeli $\mathrm{MOH}$ as part of an active epidemiological investigation, based on use of anonymous data only and no medical intervention.

The temporal changes in weekly numbers of several clinical measures were analyzed, including positive COVID-19 cases, percentage of positive tests, hospitalized patients and hospitalized patients in a severe state. COVID-19 cases were identified by a positive SARS-CoV-2 PCR test. Classification of the hospitalization severity was based on the following clinical criteria, applied on 13 July 2020 by the Israeli $\mathrm{MOH}^{24}$ based on National Institutes of Health ${ }^{25}$ and World Health Organization ${ }^{26}$ definitions: Mild illness-individuals who have any of the various signs and symptoms of COVID-19 (for example: fever, cough, malaise and loss of taste or smell); Moderate illness-individuals who have evidence of pneumonia by a clinical assessment or imaging; Severe illness-individuals who have a respiratory rate $>30$ breaths per minute, $\mathrm{SpO}_{2}<93 \%$ on room air at sea level or ratio of arterial partial pressure of oxygen to fraction of inspired oxygen $\left(\mathrm{PaO}_{2} / \mathrm{FiO}_{2}\right)<300 \mathrm{mmHg}$; and Ventilated/Critical-individuals with respiratory failure who require ventilation (invasive or non-invasive) or who have multi-organ dysfunction or shock. In this study, we denoted all patients in a severe case or worse as severe (including ventilated and critical patients).

To distinguish the effect of the vaccination campaign from other factors that might influence COVID-19 morbidity and mortality, including a third lockdown imposed in Israel during this time period, the following comparisons of the dynamic of the clinical measures mentioned above were performed. First, we compared between individuals aged 60 years and older, who were the population prioritized to receive the vaccine earlier, and younger individuals, who were prioritized according to $\mathrm{MOH}$ guidelines (Fig. 2). Second, we compared between the decline in the number of cases and hospitalizations observed after the initiation of the second national lockdown imposed by the Israeli government on 18 September 2020, with the decline observed after the initiation of the third national lockdown, imposed while the vaccination program was in place, on 8 January 2021 (Fig. 3).

Third, cities with a high percentage of individuals who were vaccinated early were compared to cities with a low percentage. For every city with more than 5,000 residents aged 60 years and older, the percentage of these individuals who received the first dose of the vaccine was calculated. Early-vaccinated cities were defined as the top ten cities with the highest percentage of individuals older than 60 years who were either vaccinated or recovered from COVID-19 by 10 January 2021 (ten cities with a total population of 641,276). Late-vaccinated cities were defined as the ten bottom cities with the lowest percentage of individuals older than 60 years who were vaccinated by 10 January 2021 (ten cities with a total population of $1,845,759$ ) (Extended Data Fig. 9)

Finally, data with a higher geographical resolution, termed GSAs, were analyzed. The GSAs are small, relatively homogeneous intra-city units defined by the Israeli Bureau of Statistics, with an average of 3,000 residents, within cities with more than 10,000 inhabitants. Data on vaccination for the GSAs were available without age group separation, although the national vaccination policy described earlier still holds information regarding the timing of each age group's vaccination. Out of 1,385 GSAs, 1,148 had more than 500 residents aged 60 years and older. Out of these 1,148 GSAs, the 400 with the highest vaccination rate by 10 January 2021 ( 3 weeks after the beginning of the vaccine drive) were denoted as early-vaccinated
GSAs and the 400 with the lowest vaccination rate by 10 January 2021 were denoted as late-vaccinated GSAs.

Reporting Summary. Further information on research design is available in the Nature Research Reporting Summary linked to this article.

\section{Data availability}

The data that support the findings of this study originate from the Israeli Ministry of Health.

National age group-level vaccination data: https://data.gov.il/dataset/covid-19/ resource/57410611-936c-49a6-ac3c-838171055b1f. Aggregated town-level and age group vaccination data are available at https://data.gov.il/dataset/covid19/resource/12c9045c-1bf4-478a-a9e1-1e876cc2e182. Aggregated GSA-level infection, hospitalization and vaccination data are available at https://data.gov.il/ dataset/covid-19/resource/d07c0771-01a8-43b2-96cc-c6154e7fa9bd. Case and hospitalization data are derived from confidential individual data and are not publicly available.

\section{Code availability}

Source code is available at https://github.com/hrossman/Patterns-of-covid19-pandemic-dynamics-following-deployment-of-a-broad-nati onal-immunization-program. All analyses were performed using the statistical Python version 3.6 and matplotlib 3.10 .

\section{References}

23. Wajnberg, A. et al. Robust neutralizing antibodies to SARS-CoV-2 infection persist for months. Science 370, 1227-1230 (2020).

24. Ministry of Health. Uniform definition of the severity of the disease in hospitalized patients with COVID-19. https://www.gov.il/he/departments/ publications/reports/mr-294754420 (7 December 2020).

25. National Institutes of Health. COVID-19 Research. https://www.nih.gov/ coronavirus

26. World Health Organization. WHO Coronavirus Disease (COVID-19) Dashboard. https://covid19.who.int/

\section{Acknowledgements}

We thank M. Bruhim, M. Goldberg and E. Sitbon for their contributions to our efforts H.R. is supported by the Israeli Council for Higher Education via the Weizmann Data Science Research Center and by a research grant from Madame Olga Klein-Astrachan.

\section{Author contributions}

H.R. conceived the project, designed and conducted the analyses, interpreted the results and wrote the manuscript. S.S. and T.M. designed and conducted the analyses, interpreted the results and wrote the manuscript. M.G., U.S. and E.S. designed the analyses, interpreted the results, wrote the manuscript and supervised and conceived the project.

\section{Competing interests}

The authors declare no competing interests.

\section{Additional information}

Extended data is available for this paper at https://doi.org/10.1038/s41591-021-01337-2. Supplementary information The online version contains supplementary material available at https://doi.org/10.1038/s41591-021-01337-2.

Correspondence and requests for materials should be addressed to M.G., U.S. or E.S.

Peer review information Nature Medicine thanks the anonymous reviewers for their contribution to the peer review of this work. Jennifer Sargent was the primary editor on this article and managed its editorial process and peer review in collaboration with the rest of the editorial team.

Reprints and permissions information is available at www.nature.com/reprints. 

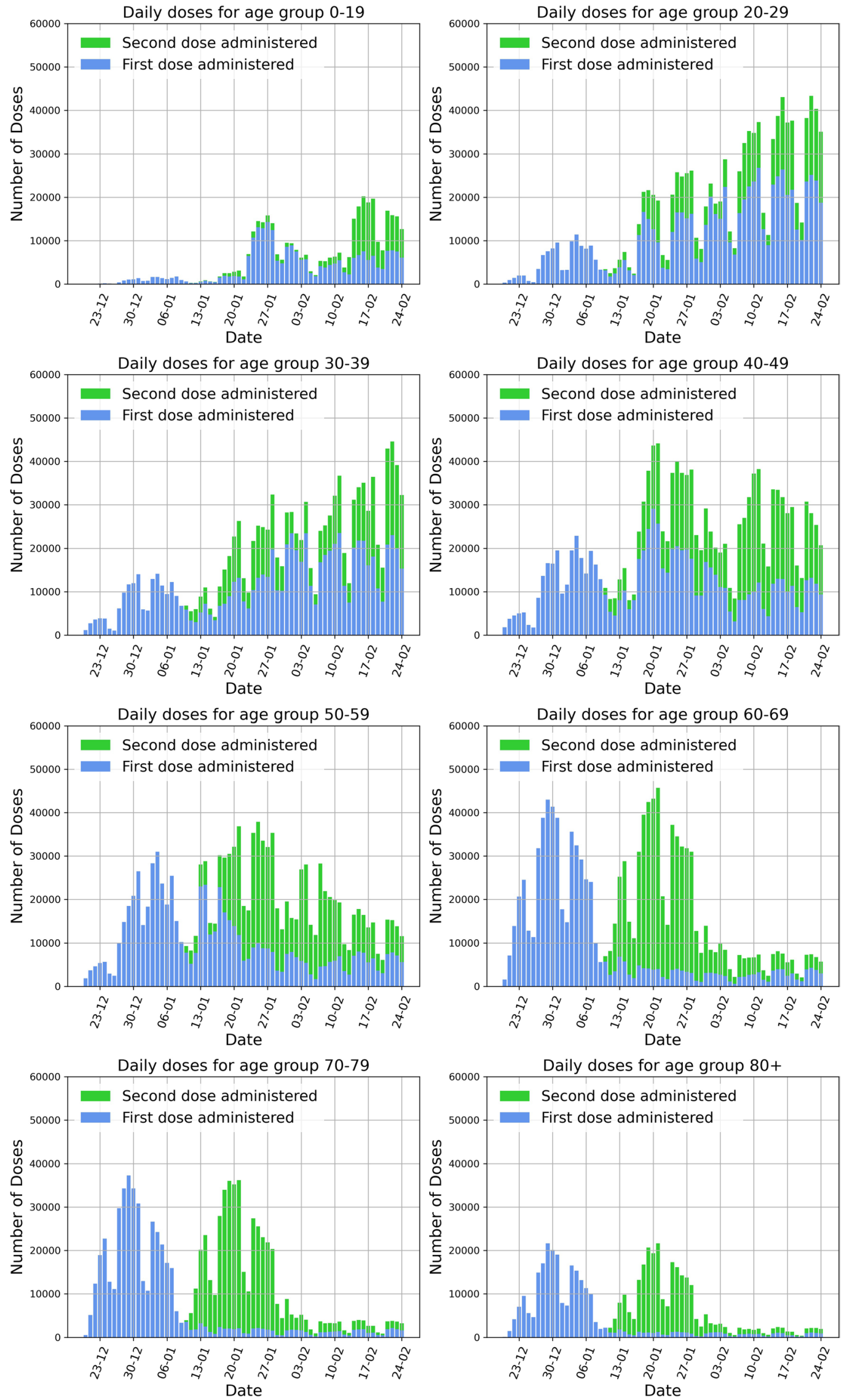

Extended Data Fig. 1 | Daily number of administered vaccination doses by age groups. Daily number of administered vaccination doses by age groups, first dose (blue bars) and second dose (green bars). 

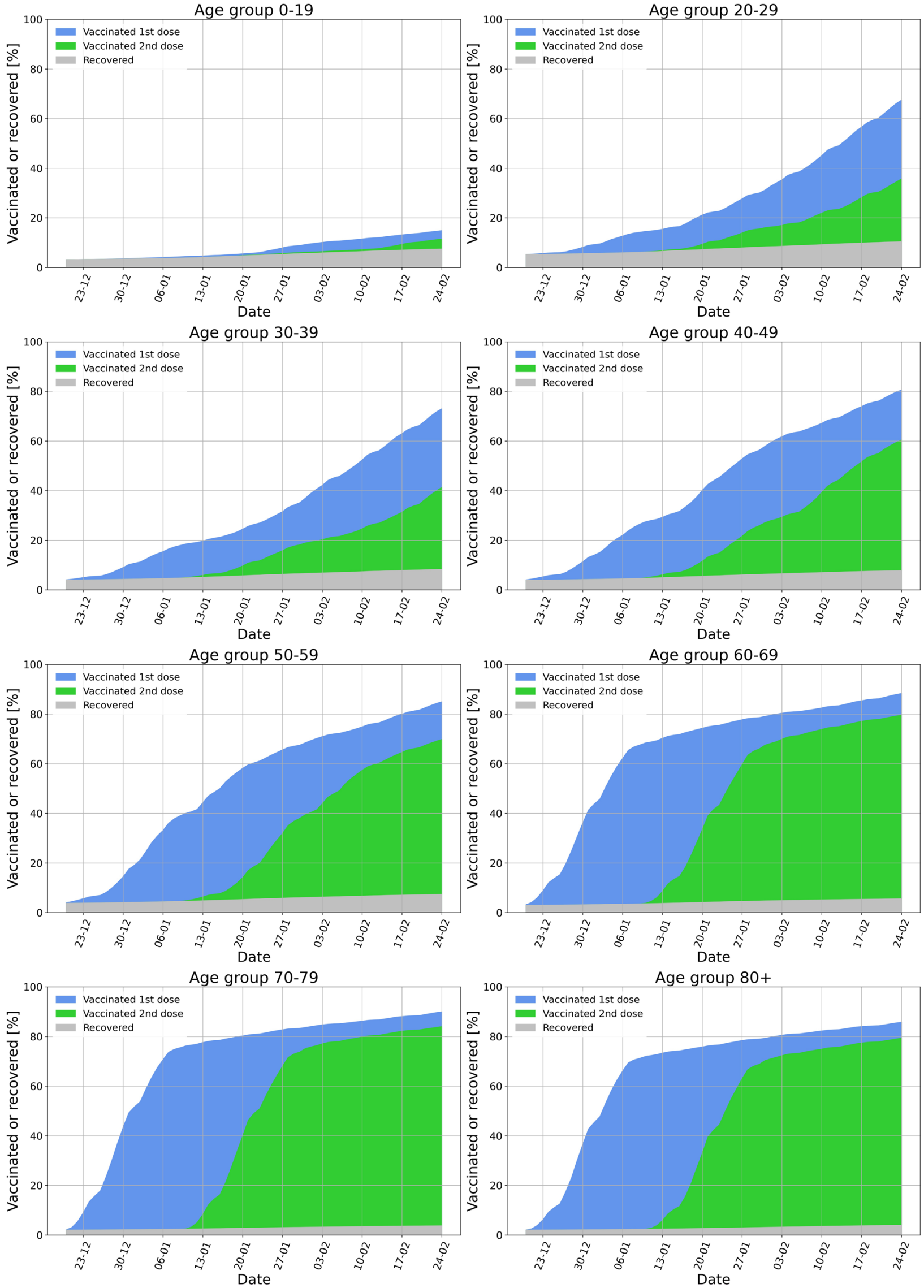

Extended Data Fig. 2 | Cumulative percentage of each age-group recovered or vaccinated. Cumulative percentage of each age-group recovered or vaccinated. Vaccinated population that received the first dose is shown in blue; and the vaccinated population that received the second dose is shown in green. Recovered population is shown in gray. 


\section{NATURE MEDICINE}

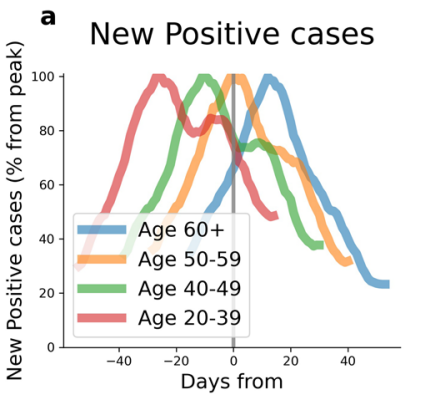

$50 \%$ of age group vaccinated 1 st dose

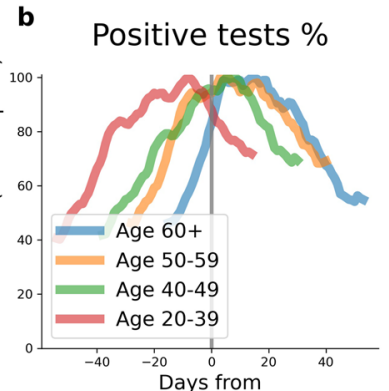

$50 \%$ of age group vaccinated 1 st dose

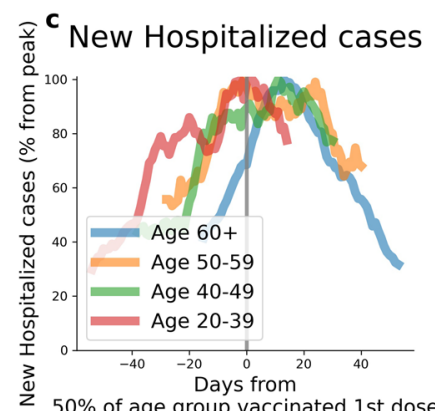

$50 \%$ of age group vaccinated 1 st dose

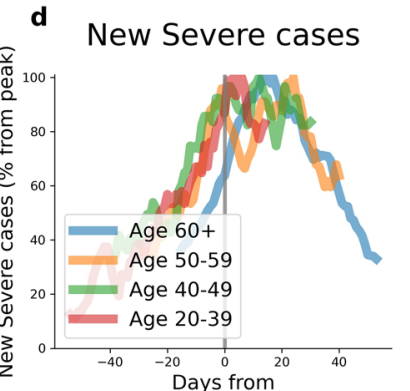

$50 \%$ of age group vaccinated 1st dose

Extended Data Fig. 3 | Comparison between age groups centered at $\mathbf{5 0 \%}$ received first dose of vaccine or recovered. Comparison between age groups 20-39 (red), 40-49 (green), 50-59 (orange) and over 60 (blue) years old in $\mathbf{a}$, Percent drop of new positive cases from peak value $\mathbf{b}$, Percent from the peak of PCR tests positive results percentage $\mathbf{c}$, Percent from the peak of new hospitalizations d, Percent from the peak of new severe cases. All curves are centered at the date in which $50 \%$ of the age group population has received the first dose or recovered. 

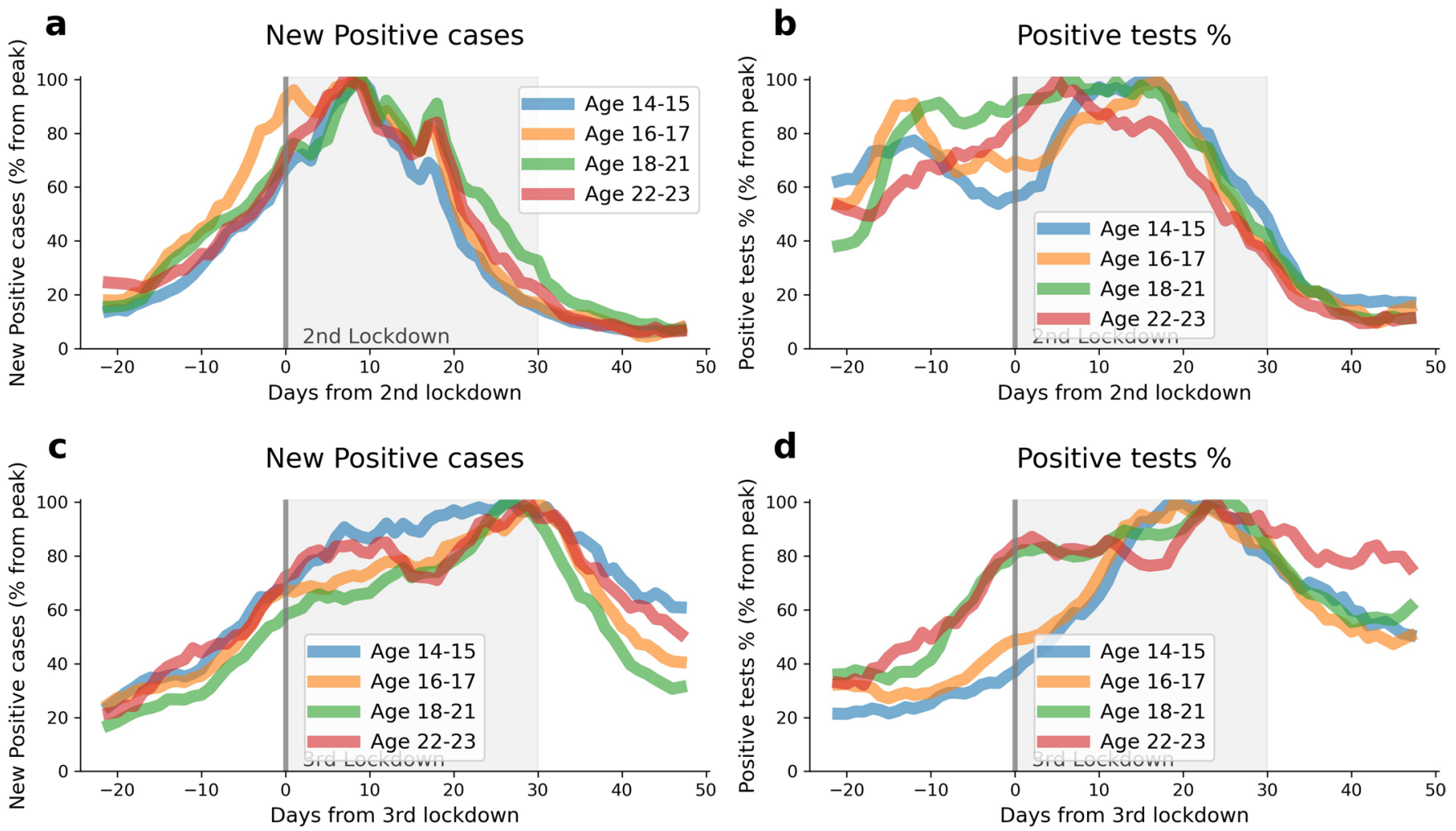

Extended Data Fig. 4 | Teenagers vaccination comparison. Comparison between age groups 14-15 (blue), 16-17 (orange), 18-21 (green) and 22-23 (red) years old in a, Percent drop of new positive cases from peak value at the time period around the second lockdown $\mathbf{b}$, Percent from the peak of PCR tests positive results percentage at the time period around the second lockdown $\mathbf{c}$, Percent drop of new positive cases from peak value at the time period around the third lockdown $\mathbf{d}$, Percent from the peak of PCR tests positive results percentage at the time period around the third lockdown. In $\mathbf{a}, \mathbf{b}$ "Day 0 " represents the second lockdown start date, September 18th 2020. In c, d "Day 0" represents the third lockdown start date, January 8th 2021. In all figures the lockdown time period is displayed as gray filling. 
Early vaccinated

a geographical statistical areas

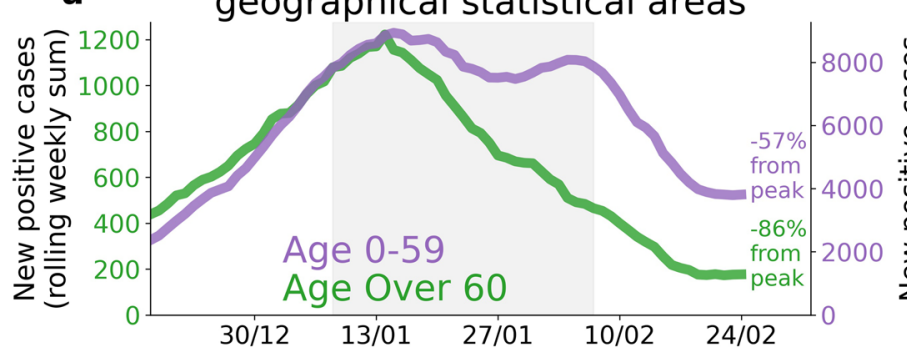

C

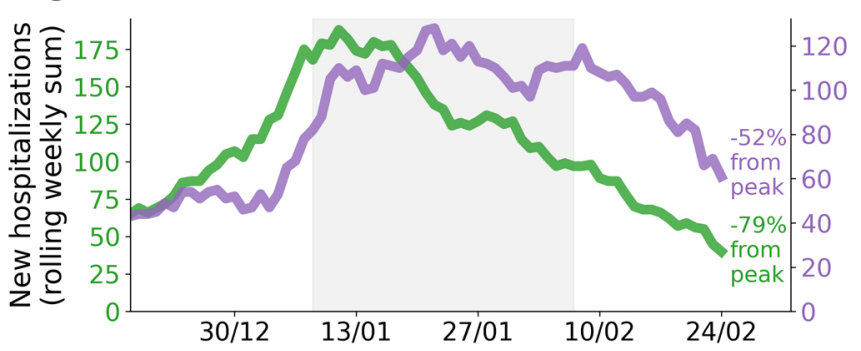

e

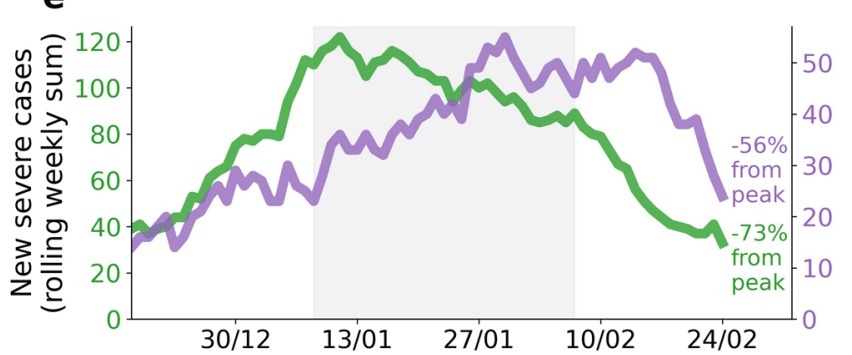

g Early vaccinated

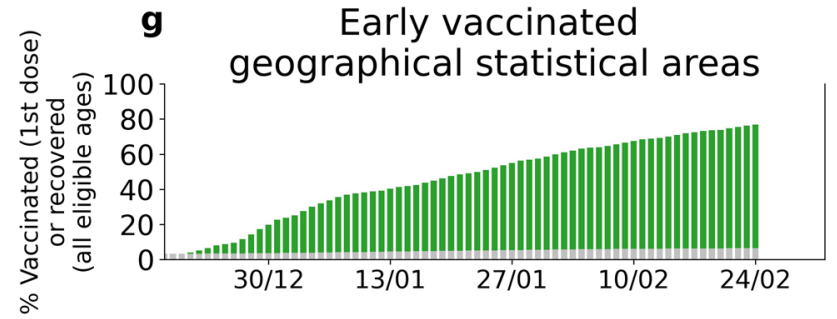

b Late vaccinated

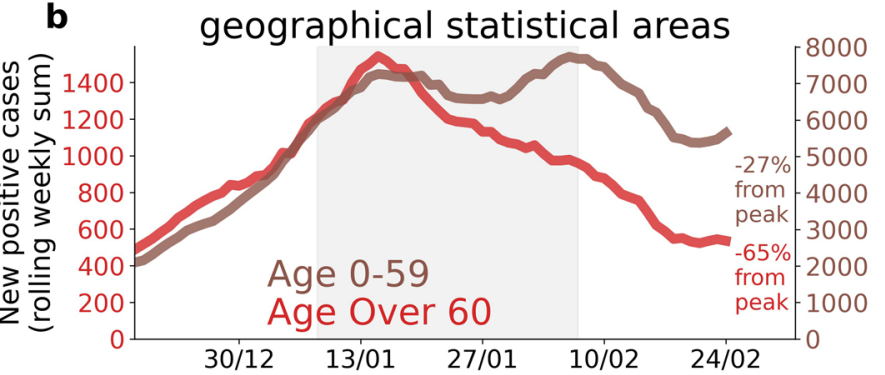

d

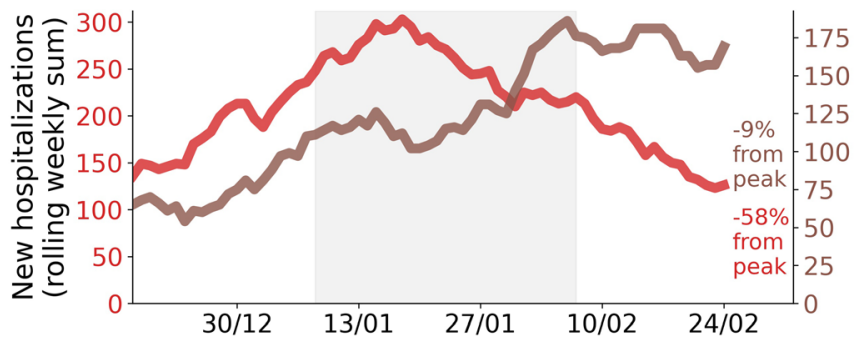

f
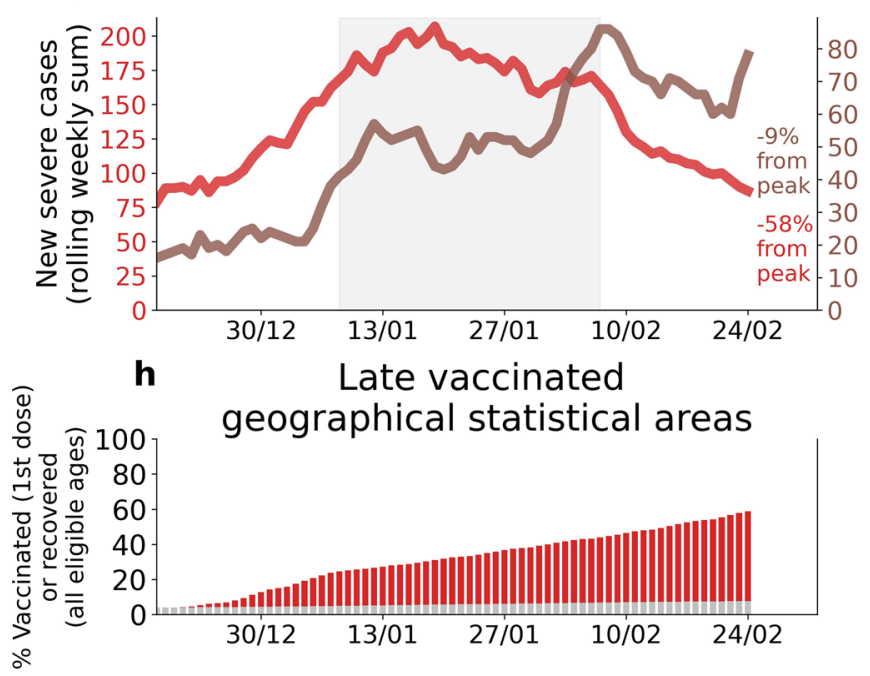

Extended Data Fig. 5 | Early-vaccinated and late-vaccinated GSAs comparison. Comparison between age groups 0-59 years old and 60+ years old from early- and late-vaccinated GSAs. In all figures the third lockdown time period is shown as gray filling. Note: Figures a-f are presented with different $y$-axis scales in order to highlight relative within-population trends. Age group 0-59 is shown as a purple line in a,c,e and as a brown line in b,d,f. Age group $60+$ is shown as a green line in $a, c, e$ and as a red line in b,d,f. a, Rolling weekly sum of new positive cases in early-vaccinated GSAs. $\mathbf{b}$, Rolling weekly sum of new positive cases in late-vaccinated GSAs. c, Rolling weekly sum of new mild, moderate or severe hospitalizations in early-vaccinated GSAs. d, Rolling weekly sum of new mild, moderate or severe hospitalizations in late-vaccinated GSAs. e, Rolling weekly sum of new severe hospitalizations in early-vaccinated GSAs. f, Rolling weekly sum of new severe hospitalizations in late-vaccinated GSAs. $\mathbf{g}$, Percentage of individuals vaccinated or recovered in time in early-vaccinated GSAs. h, Percentage of individuals vaccinated or recovered in time in late-vaccinated GSAs. A summary of this figure is shown in Supplementary Table 3. 
a

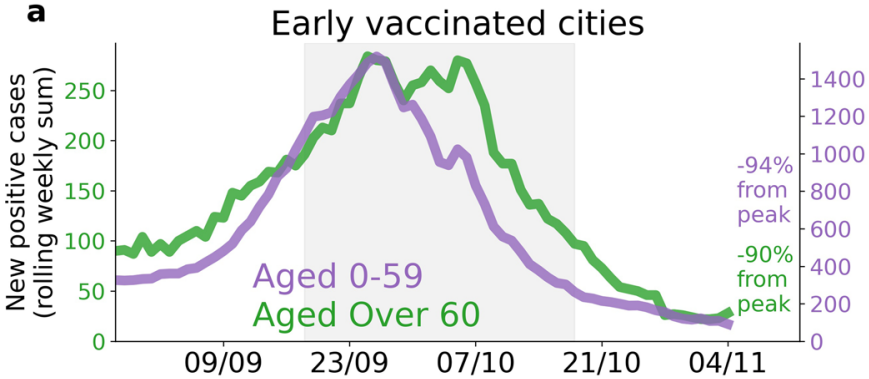

c

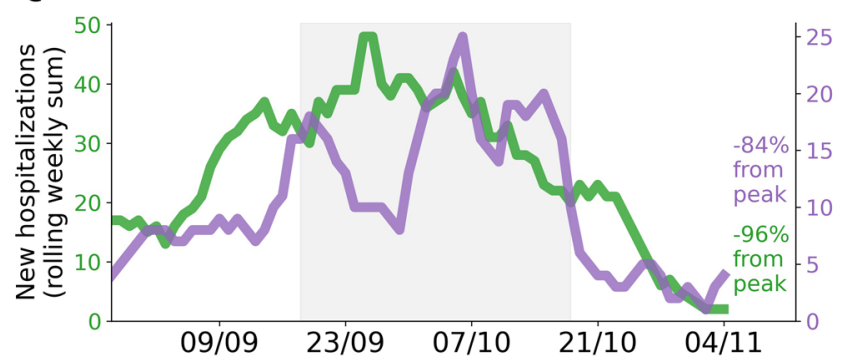

e

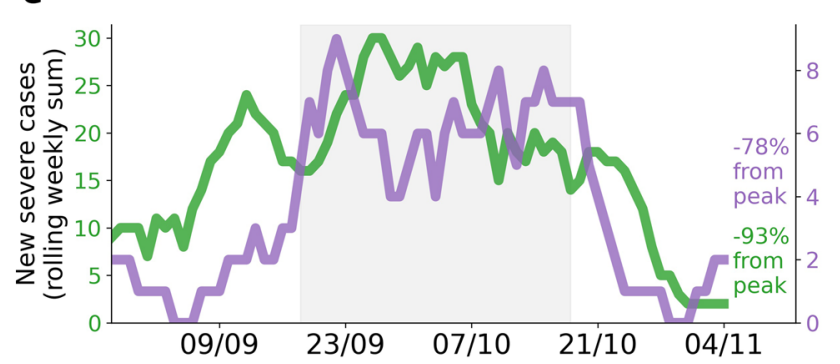

\section{b}

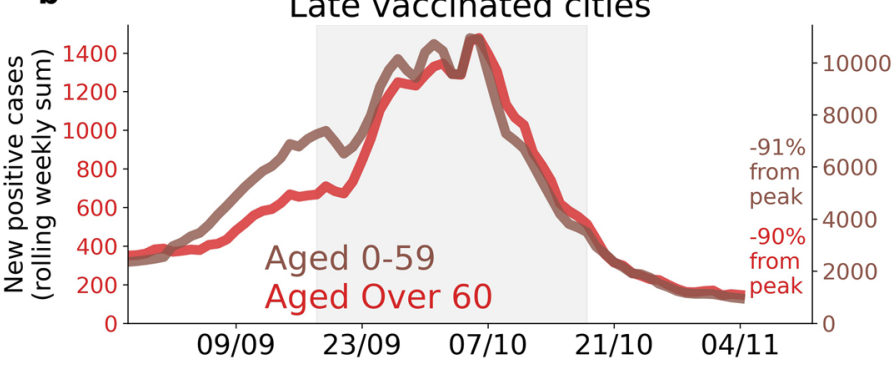

d

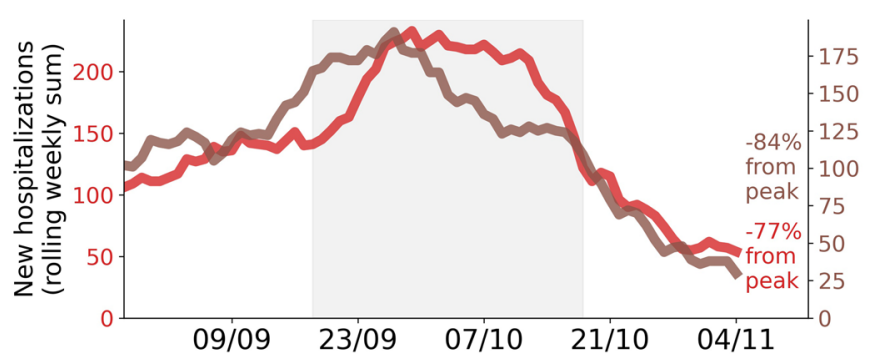

f

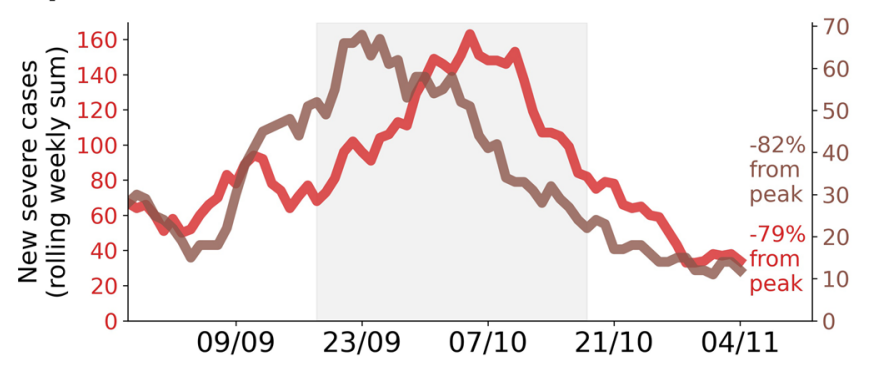

Extended Data Fig. 6 | Early-vaccinated and late-vaccinated cities comparison around September lockdown. Comparison between age groups 0-59 years old and $60+$ years old from cities with most of the population vaccinated early and cities with most of the population vaccinated late. In all figures the lockdown time period is shown as gray filling. Note: Figures a-f are presented with different $y$-axis scales in order to highlight relative within-population trends. Age group 0-59 is shown as a purple line in a,c,e and as a brown line in b,d,f. Age group 60+ is shown as a green line in a,c,e and as a red line in $b, d, f$, a, Rolling weekly sum of new positive cases in early-vaccinated cities. b, Rolling weekly sum of new positive cases in late-vaccinated cities. c, Rolling weekly sum of new mild, moderate or severe hospitalizations in early-vaccinated cities. d, Rolling weekly sum of new mild, moderate or severe hospitalizations in late-vaccinated cities. e, Rolling weekly sum of new severe hospitalizations in early-vaccinated cities. f, Rolling weekly sum of new severe hospitalizations in late-vaccinated cities. 


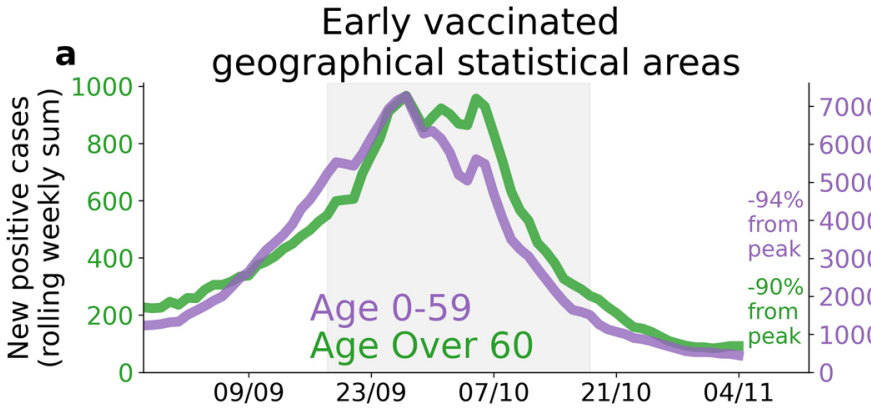

C

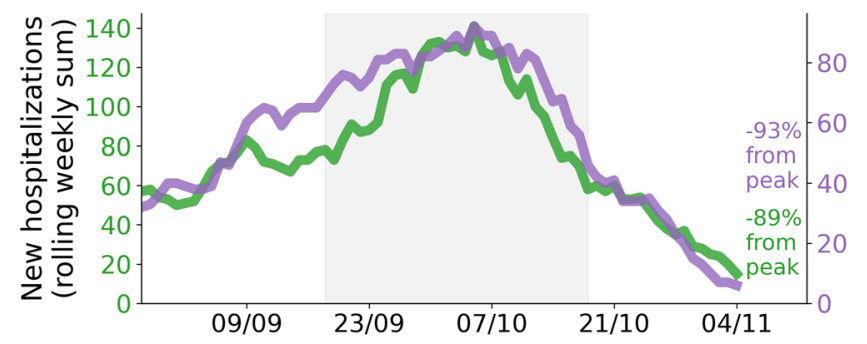

e

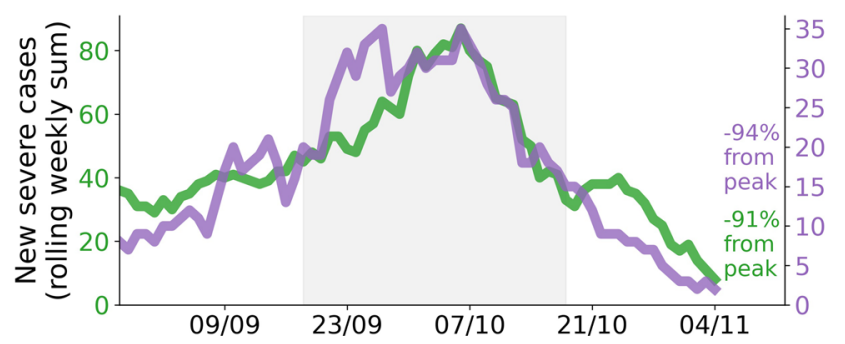

b

Late vaccinated

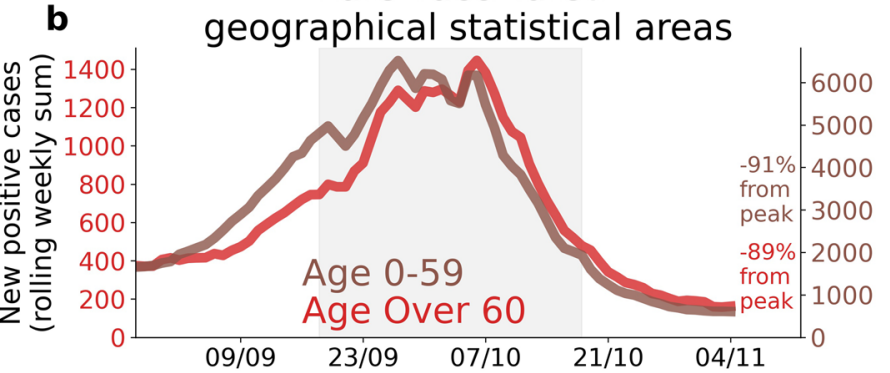

d

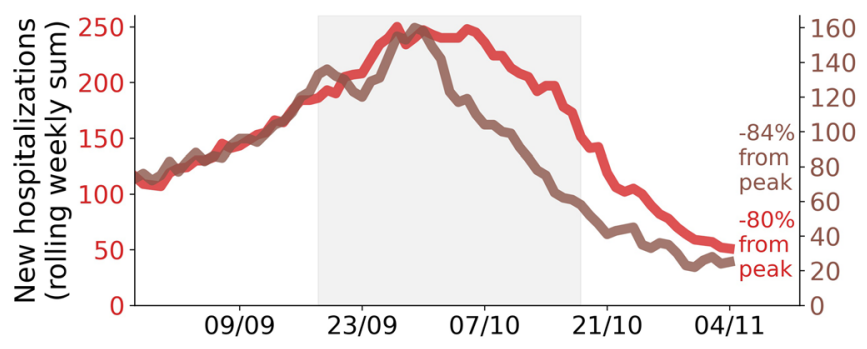

f

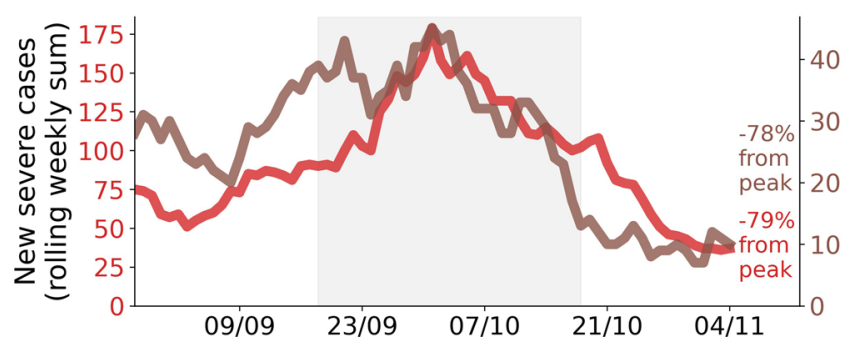

Extended Data Fig. 7 | Early-vaccinated and late-vaccinated GSAs comparison around September lockdown. Comparison between age groups 0-59 years old and 60+ years old from geographical statistical areas (GSAs) with most of the population vaccinated early and GSAs with most of the population vaccinated late. In all figures the second lockdown is shown as gray filling. Note: Figures a-f are presented with different $y$-axis scales in order to highlight relative within-population trends. Age group 0-59 is shown as a purple line in a,c,e and as a brown line in b,d,f. Age group 60+ is shown as a green line in $a, c, e$ and as a red line in $b, d, f$. $\mathbf{a}$, Rolling weekly sum of new positive cases in early-vaccinated GSAs. $\mathbf{b}$, Rolling weekly sum of new positive cases in late-vaccinated GSAs. c, Rolling weekly sum of new mild, moderate or severe hospitalizations in early-vaccinated GSAs. d, Rolling weekly sum of new mild, moderate or severe hospitalizations in late-vaccinated GSAs. e, Rolling weekly sum of new severe hospitalizations in early-vaccinated GSAs.

f, Rolling weekly sum of new severe hospitalizations in late-vaccinated GSAs. 


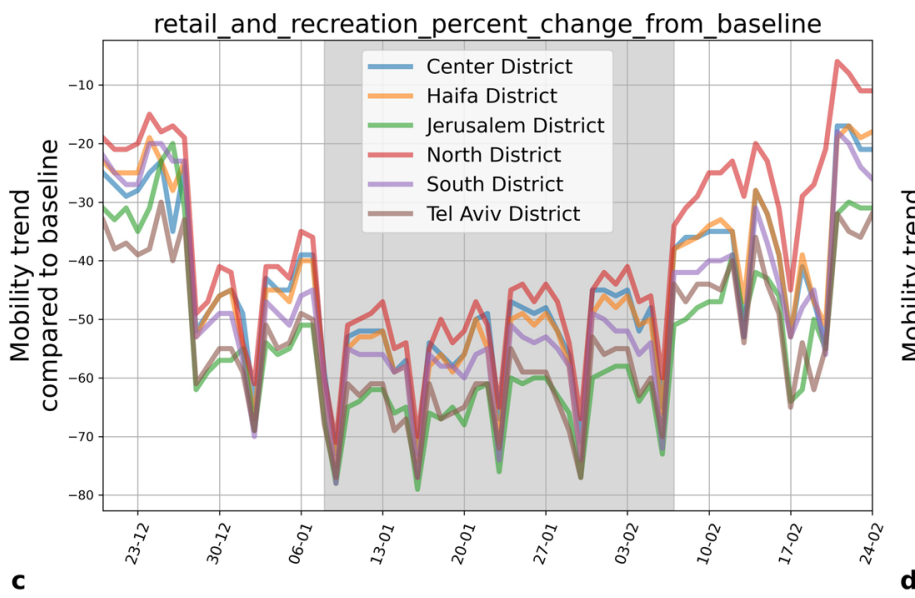

b

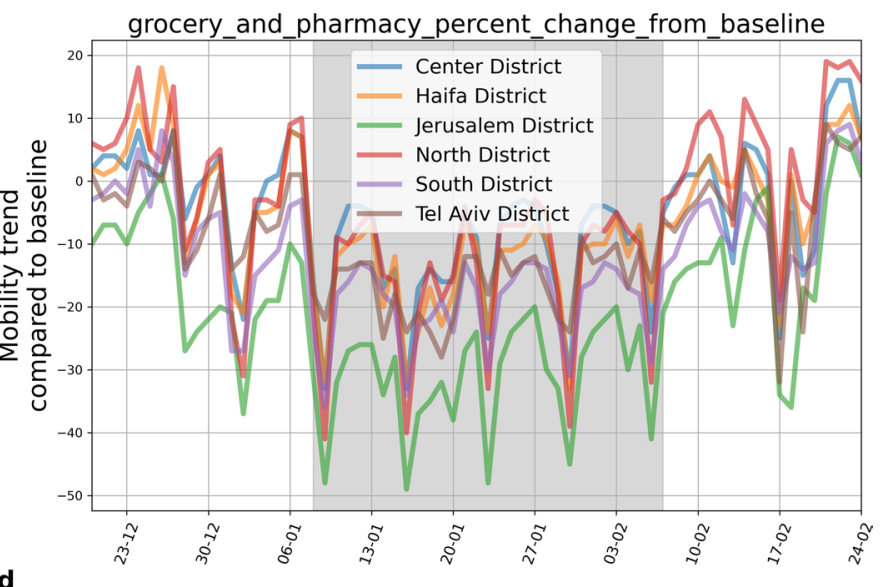

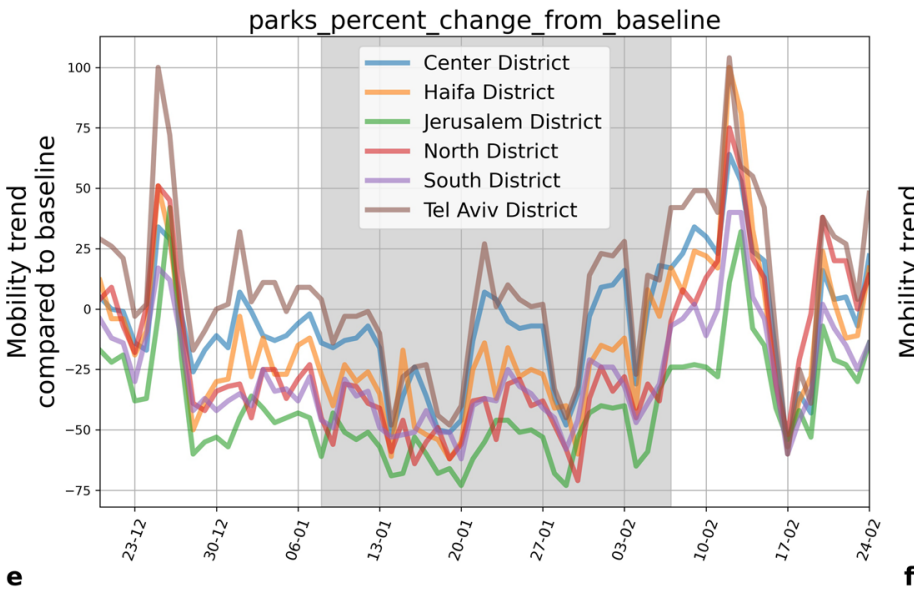
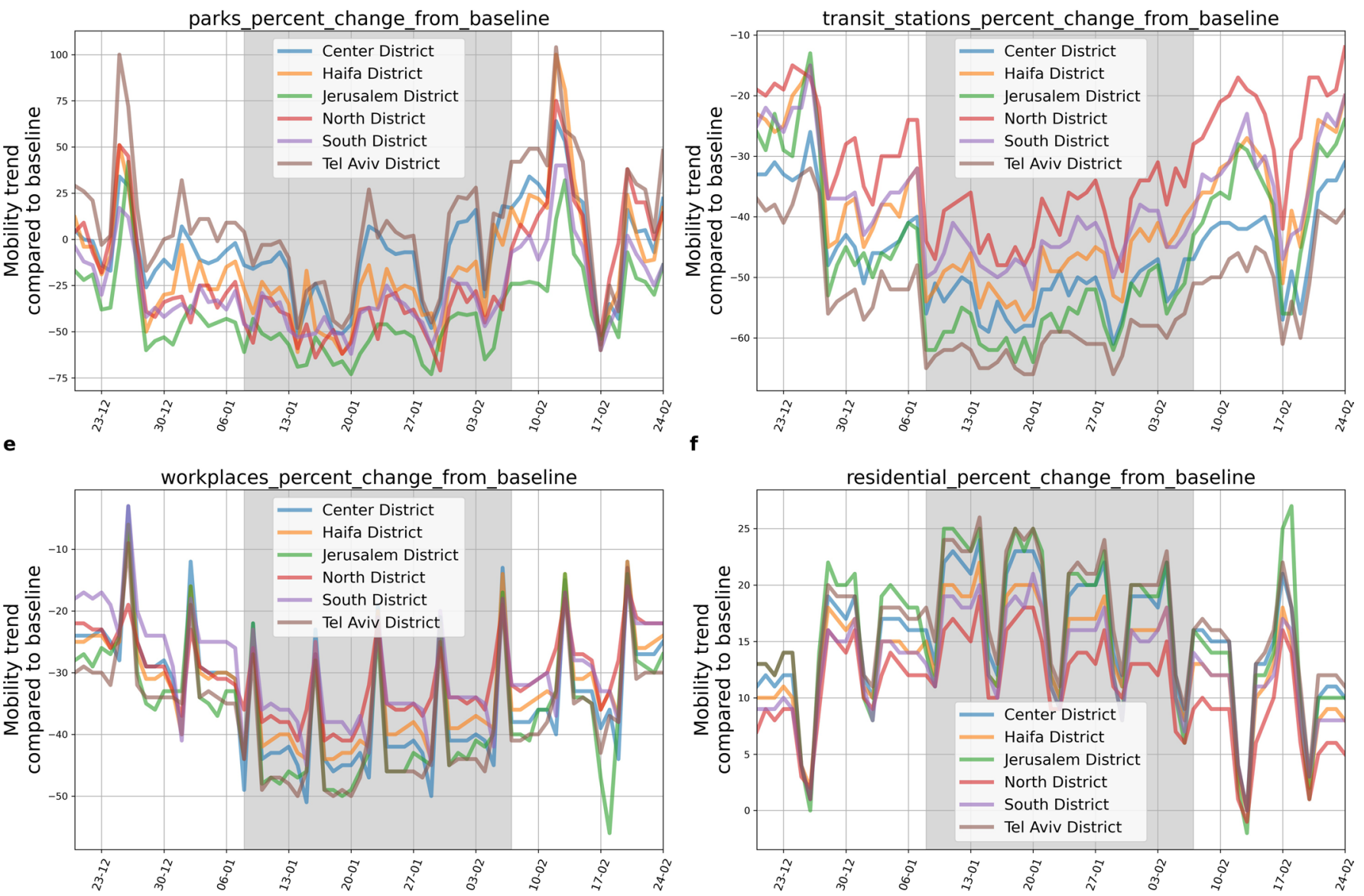

Extended Data Fig. 8 | Israel mobility data. Israel mobility data (See https://www.google.com/covid19/mobility). Mobility trend per district compared to baseline mobility, which is defined as the mobility in the date in which the vaccination campaign was initiated. In all panels, the time period of the lockdown is shown as gray filling. $\mathbf{a}$, retail and recreation mobility. $\mathbf{b}$, grocery and pharmacy mobility. $\mathbf{c}$, parks mobility. $\mathbf{d}$, transit stations mobility. e, workplaces mobility. f, residential mobility. 


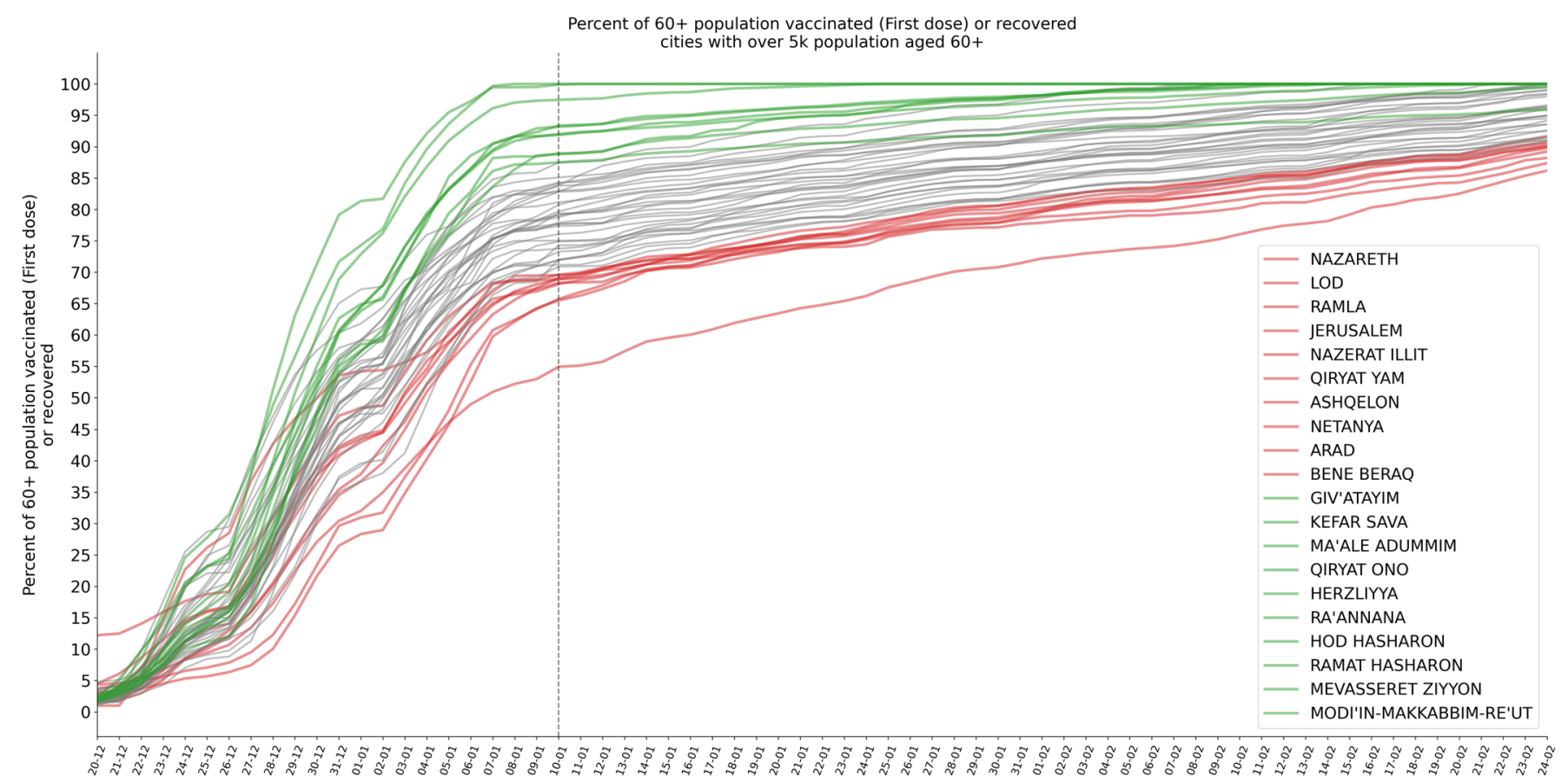

Extended Data Fig. 9 | Cumulative percentage of vaccinated population from age group 60+, in cities with more than 5,000 residents in this age group. Cumulative percentage of vaccinated population from age group 60+, in cities with more than 5,000 residents in this age group. Early-vaccinated cities are shown as green lines, late-vaccinated cities are shown as red lines. Other cities are shown as gray lines. 


\section{Reporting Summary}

Nature Research wishes to improve the reproducibility of the work that we publish. This form provides structure for consistency and transparency in reporting. For further information on Nature Research policies, see our Editorial Policies and the Editorial Policy Checklist.

\section{Statistics}

For all statistical analyses, confirm that the following items are present in the figure legend, table legend, main text, or Methods section.

n/a Confirmed

$\square$ The exact sample size $(n)$ for each experimental group/condition, given as a discrete number and unit of measurement

$\square$ \A statement on whether measurements were taken from distinct samples or whether the same sample was measured repeatedly

$\triangle$ The statistical test(s) used AND whether they are one- or two-sided

Only common tests should be described solely by name; describe more complex techniques in the Methods section.

$\bigotimes$ A description of all covariates tested

$\square$ \A description of any assumptions or corrections, such as tests of normality and adjustment for multiple comparisons

$\square$ A full description of the statistical parameters including central tendency (e.g. means) or other basic estimates (e.g. regression coefficient)

$\bigotimes$ AND variation (e.g. standard deviation) or associated estimates of uncertainty (e.g. confidence intervals)

$\bigotimes \square \begin{aligned} & \text { For null hypothesis testing, the test statistic (e.g. } F, t, r \text { ) with confidence intervals, effect sizes, degrees of freedom and } P \text { value noted } \\ & \text { Give } P \text { values as exact values whenever suitable. }\end{aligned}$

Х $\square$ For Bayesian analysis, information on the choice of priors and Markov chain Monte Carlo settings

Х $\square$ For hierarchical and complex designs, identification of the appropriate level for tests and full reporting of outcomes

Х $\square$ Estimates of effect sizes (e.g. Cohen's $d$, Pearson's $r$ ), indicating how they were calculated

Our web collection on statistics for biologists contains articles on many of the points above.

\section{Software and code}

Policy information about availability of computer code

Data collection Python version 3.6

Data analysis Python version 3.6, Matplotlib 3.1.0

For manuscripts utilizing custom algorithms or software that are central to the research but not yet described in published literature, software must be made available to editors and reviewers. We strongly encourage code deposition in a community repository (e.g. GitHub). See the Nature Research guidelines for submitting code \& software for further information.

\section{Data}

Policy information about availability of data

All manuscripts must include a data availability statement. This statement should provide the following information, where applicable:

- Accession codes, unique identifiers, or web links for publicly available datasets

- A list of figures that have associated raw data

- A description of any restrictions on data availability

The data that support the findings of this study originates from the Israel Ministry of Health. National age-group level vaccination data:

https://data.gov.il/dataset/covid-19/resource/57410611-936c-49a6-ac3c-838171055b1f

Aggregated town-level and age groups vaccination data is available at:

https://data.gov.il/dataset/covid-19/resource/12c9045c-1bf4-478a-a9e1-1e876cc2e182

Aggregated GSA -level infection, hospitalization and vaccination data is available at:

https://data.gov.il/dataset/covid-19/resource/d07c0771-01a8-43b2-96cc-c6154e7fa9bd

Cases and hospitalizations data are derived from confidential individual data and are not publicly available. 


\section{Field-specific reporting}

Please select the one below that is the best fit for your research. If you are not sure, read the appropriate sections before making your selection.

\Life sciences

$\square$ Behavioural \& social sciences

Ecological, evolutionary \& environmental sciences

For a reference copy of the document with all sections, see nature.com/documents/nr-reporting-summary-flat.pdf

\section{Life sciences study design}

All studies must disclose on these points even when the disclosure is negative.

Sample size The sample includes all positive PCR COVID-19 patients in Israel between 1/03/2020 to 24/02/2021
Data exclusions No data was excluded
Replication Randomization is not applicable to this study.
Randomization
Blinding Blinding is not applicable to this study, as this is a retrospective observational study.

\section{Reporting for specific materials, systems and methods}

We require information from authors about some types of materials, experimental systems and methods used in many studies. Here, indicate whether each material, system or method listed is relevant to your study. If you are not sure if a list item applies to your research, read the appropriate section before selecting a response.

Materials \& experimental systems

\begin{tabular}{l|l}
\hline $\mathrm{n} / \mathrm{a}$ & Involved in the study \\
$\square$ & $\square$ Antibodies \\
$\square$ & $\square$ Eukaryotic cell lines \\
$\searrow$ & $\square$ Animals and other organisms \\
$\square$ & $\square$ Human research participants \\
$\square$ & $\square$ Clinical data \\
$\square$ & $\square$ Dual use research of concern
\end{tabular}

\begin{tabular}{l|l}
\multicolumn{2}{l}{ Methods } \\
\hline n/a & Involved in the study \\
$\square$ & $\square$ ChIP-seq \\
$\searrow$ & $\square$ Flow cytometry \\
$\searrow$ & $\square$ MRI-based neuroimaging
\end{tabular}

